\title{
Low viscosity contrast fingering in a rotating Hele-Shaw cell
}

\author{
E. Alvarez-Lacalle, ${ }^{\text {a) }}$ J. Ortín, and J. Casademunt \\ Departament d'Estructura $i$ Constituents de la Matèria, Facultat de Física, Universitat de Barcelona, \\ Avinguda Diagonal 647, E-08028 Barcelona, Spain
}

(Received 11 June 2003; accepted 4 December 2003; published online 27 February 2004)

\begin{abstract}
We study the fingering instability of a circular interface between two immiscible liquids in a radial Hele-Shaw cell. The cell rotates around its vertical symmetry axis, and the instability is driven by the density difference between the two fluids. This kind of driving allows studying the interfacial dynamics in the particularly interesting case of an interface separating two liquids of comparable viscosity. An accurate experimental study of the number of fingers emerging from the instability reveals a slight but systematic dependence of the linear dispersion relation on the gap spacing. We show that this result is related to a modification of the interface boundary condition which incorporates stresses originated from normal velocity gradients. The early nonlinear regime shows nearly no competition between the outgrowing fingers, characteristic of low viscosity contrast flows. We perform experiments in a wide range of experimental parameters, under conditions of mass conservation (no injection), and characterize the resulting patterns by data collapses of two characteristic lengths: the radius of gyration of the pattern and the interface stretching. Deep in the nonlinear regime, the fingers which grow radially outwards stretch and become gradually thinner, to a point that the fingers pinch and emit drops. We show that the amount of liquid emitted in the first generation of drops is a constant independent of the experimental parameters. Further on there is a sharp reduction of the amount of liquid centrifugated, punctuated by periods of no observable centrifugation. (C) 2004 American Institute of Physics. [DOI: 10.1063/1.1644149]
\end{abstract}

\section{INTRODUCTION}

Within the broad subject of spatio-temporal pattern formation in nonequilibrium systems, ${ }^{1}$ the problems of interfacial pattern formation have attracted much attention in the last 2 decades. This class of problems admits a description in terms of an interface which separates two distinct, macroscopically structureless phases, so that the spatio-temporal evolution of the system is given directly by the temporal evolution of the morphology of the interface. Processes of this kind include fluid flow in porous media, crystal growth, chemical electrodeposition, and flame propagation. ${ }^{2}$

A prototype system of interfacial pattern formation problems is the morphological instability of the interface between two immiscible fluids confined in a Hele-Shaw cell. This system is relatively simple, both experimentally and theoretically, and yet exhibits nontrivial dynamics.

One interesting aspect of this problem is the nontrivial role of the viscosity contrast between the two fluids on the dynamics. In the channel geometry, a series of numerical simulations, ${ }^{3}$ experiments, ${ }^{4}$ and further theoretical studies ${ }^{5,6}$ has proved that the viscosity contrast plays an important dynamical role in the deeply nonlinear regime, particularly on the mechanisms of finger competition and the resulting interface morphologies. The research reported in the present paper is motivated by the question whether and how the viscosity contrast plays a similarly crucial role in the radial geometry, a question not yet addressed.

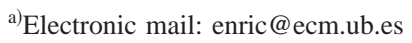

In the channel geometry, the limit of low viscosity contrast could be explored experimentally by performing gravity-driven experiments. ${ }^{4}$ In this case the instability is basically originated by the density difference (not the viscosity difference) between the two fluids. There is an exact parameter mapping between injection- and gravity-driven flows in the channel geometry, however, which makes the two flows equivalent in a dimensionless formulation, in the reference frame moving with the average velocity of the interface.

The situation in the radial geometry is more complex, since there is no simple analog of gravity in this geometry. One interesting candidate is centrifugal driving, produced by rotating the circular cell around its vertical symmetry axis. Under centrifugal driving the instability also originates basically from the density difference between the two fluids, as in the gravity-driven case. Centrifugally-driven Hele-Shaw flows, however, cannot be exactly mapped to injectiondriven flows (and hence are not the exact counterparts of gravity-driven experiments, in the radial geometry). ${ }^{7}$

Hele-Shaw flows under rotation have been recently the subject of several theoretical and experimental studies. The linear stability analysis of an axisymmetric drop in the case of high density and high viscosity contrast, originally worked out by Schwartz, ${ }^{8}$ has been extended to arbitrary density and viscosity contrast by Carrillo et al. ${ }^{9}$ There have also been a number of theoretical efforts to find families of exact solutions for interfaces evolving out of an axisymmetric drop ${ }^{7,10}$ or an axisymmetric annular configuration ${ }^{11}$ under rotation. Recently, it has been shown that rotation can help preventing 
the formation of cusp singularities in problems with zero surface tension. ${ }^{12}$

Regarding experimental work, our group has pioneered the study of interfacial instabilities under centrifugal forcing, investigating the instabilities of axisymmetric drops in a rotating Hele-Shaw cell, in the limit of high viscosity contrast. ${ }^{9}$ Specifically, we have verified the maximum growth rate selection of initial patterns with and without injection, and also studied the nonlinear regime in the case of vanishing injection rate. A second series of experiments in the rotating cell has focused on the dynamics of axisymmetric annular configurations, with air at the innermost and outermost layers, and oil at the intermediate layer. ${ }^{13}$ This arrangement leads to a rich variety of patterns, as the density difference drives the instability of the leading interface (oil displacing air) and the viscosity difference drives the instability of the trailing interface (air displacing oil). We have proved that the stability of the two interfaces is coupled through the pressure field already at a linear level. By performing experiments in prewet and dry conditions, we have shown that the stability of the interfaces depends substantially on the wetting conditions at the leading interface.

The main objective of this paper is to extend our previous experimental studies in the rotating Hele-Shaw cell to low viscosity contrast flows. Specifically, we report on a detailed experimental investigation of the linear and deeply nonlinear evolution of a circular axisymmetric interface separating two immiscible liquids. We focus on the case of vanishing injection rate, $Q=0$, which is the closest analog to a gravity-driven experiment. This simplification reduces the number of independent parameters in the problem to two: namely $S$ (ratio of centrifugal to capillary forces) and $A$ (viscosity contrast or Atwood ratio), and we expect data collapse of several magnitudes to simple scaling laws more feasible. The other experimental parameters (fluid volume, rotational frequency, and gap thickness) are modified in a wide range of values.

Experiments of low viscosity contrast flows in the rotating cell lead to interesting dynamical and morphological effects in the highly nonlinear regime. In particular, we report on the frequent ocurrence of pinch-off singularities of the radially growing fingers, and the concomitant emission of droplets. Such phenomena were also observed in low viscosity contrast gravity-driven experiments in the channel geometry. ${ }^{4}$

The outline of the paper is as follows: Section II provides details of the experimental setup, the physical properties of the fluids, and the experimental procedure, together with a general, qualitative picture of the dynamical and morphological properties of the patterns observed in our experiments. Section III is dedicated to analyze and discuss the experimental results. First we outline the Hele-Shaw equations of the problem, with particular attention to the role of the Young-Laplace boundary condition at the interface, and compare the prediction of a linear stability analysis for the fastest growing mode against experimental results. Second, we present a quantitative characterization of the patterns, based on their latency time, the interface stretching, and the mass distribution. Next, we study pinch-off events and the properties of emitted droplets, and analyze the latest stages of the pattern evolution. This section concludes with a discussion of the role of low surface tension based on numerical simulations. Finally, in Sec. IV, we provide a summary of our main results and draw conclusions.

\section{EXPERIMENT}

\section{A. Experimental setup}

The experiments reported in this paper have been performed in a radial Hele-Shaw cell formed by two circular glass plates placed parallel one above the other, separated by a narrow gap spacing $b$. The glass plates have $390 \mathrm{~mm}$ diameter and either 7 or $10 \mathrm{~mm}$ thickness, and present a maximum deviation of $\pm 0.03 \mathrm{~mm}$ from perfect flatness. The narrow spacing between the plates is provided either by a tefflon ring or by six metallic spacers, placed near the edge of the plates. The cell is placed on a sturdy rotating platform driven by a dc motor and reductor.

The initial condition consists on an outer layer of vaseline oil (fluid 1) and an inner layer of colored silicone oil (fluid 2), separated by a circular interface (radius $R_{0}$ ) centered with the vertical symmetry axis of the cell. The two oils are immiscible. The inner liquid is denser $\left(\rho_{2}>\rho_{1}\right)$ and more viscous $\left(\mu_{2}>\mu_{1}\right)$ than the outer liquid. The cell is closed, i.e., there is no further injection or withdrawal of liquid ( $Q$ $=0$ ) during the experiment.

In the experiment the cell is set into rotation around its symmetry axis, at a prescribed angular velocity $\Omega$. The evolution of the interface is monitored with a charge coupled device camera mounted above the cell, and digitally recorded in a PC.

Additional details of the experimental setup can be found in Refs. 9 and 13.

\section{B. Physical properties of the liquids}

The vaseline oil used in the present experiments is manufactured by Panreac (Ref. 141003). The silicone oil is manufactured by Rhodorsil (Ref. 47V 500). Their nominal densities at $20^{\circ} \mathrm{C}$ are $\rho_{1}=875 \pm 10 \mathrm{~kg} / \mathrm{m}^{3}$ for the vaseline oil and $\rho_{2}=975 \pm 10 \mathrm{~kg} / \mathrm{m}^{3}$ for the silicone oil. Their dynamic viscosities have been measured at several temperatures with a concentric cylinder viscometer HAAKE RV20/CVB20N and with a Cannon-Fenske capillary viscometer. The results are independent of the technique used within 5\%, and are shown in Figs. 1 and 2.

Originally the two liquids are colorless. In order to render the interface visible, the silicone oil has been colored blue by dispersing into it a tiny amount of oil colorant manufactured by Galloplast S.L. Dynamic viscometric measurements confirm that the liquid remains Newtonian after the coloring process, and its viscosity is not significantly affected.

The interfacial tension between the two liquids, $\sigma_{0}$, is on the order of $1 \mathrm{mN} / \mathrm{m}$, typically 1 order of magnitude smaller than the surface tension at an oil-air interface. This value makes it difficult to measure $\sigma_{0}$ accurately. On the one hand, we have found it too small to be measured with the 


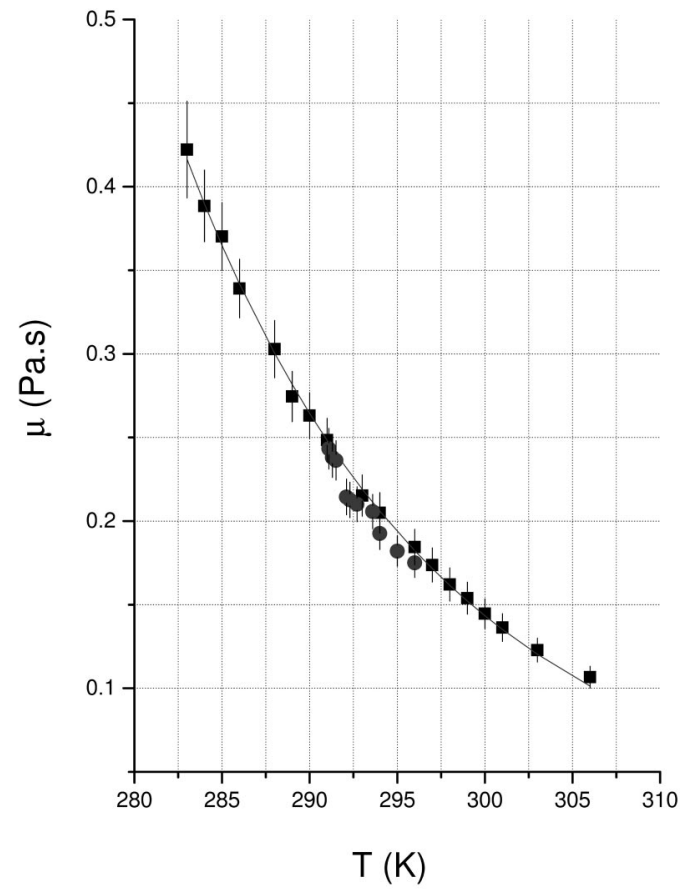

FIG. 1. Dynamic viscosity of the vaseline oil Panreac 141003 as a function of temperature. The measurements have been performed with a capillary viscometer (circles) and with a concentric cylinder viscometer (squares). The solid line is a fit of the Arrhenius equation, $\mu=P_{1} \exp \left(-E_{1} / k_{B} T\right)$, to the latter data.

classical method of the Du Noüy ring (tensiometer KRUSS K100), since the lamella formed around the ring breaks before a reliable measurement can be taken. On the other hand, this value of $\sigma_{0}$ is just at the upper measuring limit of the spinning drop technique, designed for ultralow interfacial tensions. By averaging several measurements taken with a spinning drop tensiometer KRUSS SITE104 we have been able to obtain a value $\sigma_{0}=1.8 \pm 0.7 \mathrm{mN} / \mathrm{m}$, subjected to a large experimental uncertainty.

\section{Details of the experimental procedure}

Preparation of the glass plates at the beginning of each experimental run starts by cleaning them with a soap solution, followed by rinsing with distilled water and cleaning again with acetone. This cleaning protocol is sufficient to obtain reproducible results. Occasionally we have also used a chromic mixture to complete the cleaning procedure, with no significant differences.

The initial circular interface is prepared in the following way. We inject the first liquid (vaseline oil) through an orifice at the center of the top plate, using a syringe pump, until it fills the whole cell. The injection rate is not critical in this case. We have used rates in the range from 75 to $350 \mathrm{ml} / \mathrm{h}$ with no noticeable differences. Next, we inject the second liquid (colored silicone oil) until it fills an inner circular region of the required radius $R_{0}$. Since the silicone oil is more viscous than the vaseline oil, the displacement is stable and the interface remains circular. We have found that this second liquid must be injected at a relatively high rate to ensure that a thin wetting layer of vaseline oil is left on each

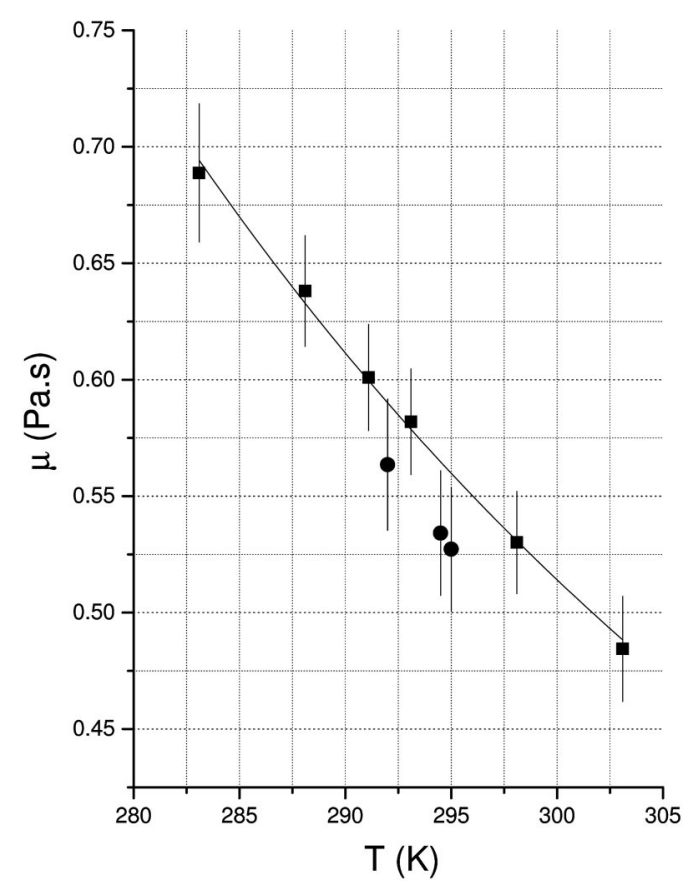

FIG. 2. Dynamic viscosity of the silicone oil Rhodorsil 47V 500, colored blue, as a function of temperature. The measurements have been performed with a capillary viscometer (circles) and with a concentric cylinder viscometer (squares). The solid line is a fit of the Arrhenius equation, $\mu$ $=P_{1} \exp \left(-E_{1} / k_{B} T\right)$, to the latter data.

glass plate. This condition ensures that advancing and receding parts of the interface will find similar wetting conditions during the experiment. Our experience is that the second liquid must be injected at rates higher than $100 \mathrm{ml} / \mathrm{h}$ in order to obtain reproducible results, with no observable differences up to $450 \mathrm{ml} / \mathrm{h}$. Typically the injection rate has been set to $400 \mathrm{ml} / \mathrm{h}$.

Once the two liquids are in the cell, the silicone oil filling the central region finds itself sandwiched between the two thin layers of vaseline oil attached to the glass plates. Because of the density difference, this stratification is gravitationally unstable for the bottom layer. Our observations show that a dripping instability develops past the first $6 \mathrm{~min}$ approximately after the generation of the initial condition, leading to the corrugation of the wetting layers of vaseline oil and, finally, to the appearance of vaseline oil drops in the silicone oil. In order to avoid any interference of this instability with our experiments, we have restricted our analysis to experiments in which the patterns studied are completely developed within 1 min after the generation of the initial condition. For this reason we have taken $\Omega=150 \mathrm{rpm}$ as a minimum rotational frequency, and $R_{0}=25 \mathrm{~mm}$ as a minimum initial radius, except for very high frequencies $(\Omega$ $>240 \mathrm{rpm}$ ) for which we can explore initial radii down to $R_{0}=15 \mathrm{~mm}$ safely.

We have taken particular care to avoid the presence of air bubbles in the cell. Air bubbles tend to form either at the circular end of the cell or at the interface between the two liquids and, when the cell is set into rotation, they move straight to the center of the cell across the interface, spoiling the experiment. In order to prevent the occurrence of air 
bubbles, the procedure for closing the cell is critical. This procedure differs depending on the type of gap spacers used.

First, we have used six metallic spacers placed approximately equispaced around the cell edge. In this case the plates are tightly clamped at these six points from the beginning. The air exits the cell freely while the liquids are being injected, until the injection of the second liquid is almost complete. Then the edge of the cell is closed with a rubber band, and the injection is interrupted. Air bubbles formed close to the rubber band leave the cell thanks to the slight overpressure created by the remaining liquid injection, which thereafter is accommodated by the rubber band itself.

The closing procedure is different when the gap spacing is provided by an annular tefflon spacer. In this case, although the glass plates are loosely clamped to keep them aligned with the axis of rotation, we leave a free space between the Teflon spacer and the top plate to evacuate the air displaced by the injection of the first liquid. Once this first liquid reaches the Teflon spacer, injection is stopped. At this point it is usual to have air bubbles formed at the contact of the liquid with the tefflon spacer. In order to remove them, the cell is tightly clamped at six points, as before, and it is set to rotate until the air bubbles reach the central hole. The bubbles are then withdrawn by producing a slight underpressure in the cell, which is compensated by the injection of the second liquid. Since this second injection must also be carried out with the cell tightly closed, to avoid the formation of new bubbles near the tefflon spacer, we restrict ourselves to initial radii in the range $20<R_{0}<50 \mathrm{~mm}$ in order to prevent a significant deformation of the glass plates. Measurements of the plate deflection with a mechanical strain-gauge show that a nominal gap spacing $b=1.2 \mathrm{~mm}$ increases by about $5 \%$ with the glass plates $10 \mathrm{~mm}$ thick, and by about $10 \%$ with the glass plates $7 \mathrm{~mm}$ thick.

Although the first closing procedure has the advantage of not producing any observable deformation of the glass plates, the second procedure is more efficient in preventing the formation of air bubbles in the cell.

Our experiments have covered a large region of the available parameter space. We have explored values of $R_{0}$ (radii of the initial circular interface) in the range from 15 to $85 \mathrm{~mm}$, and rotational frequencies $\Omega$ in the range from 150 to $300 \mathrm{rpm}$. The values selected for the gap thickness $b$ have been $0.70 \pm 0.04,1.00 \pm 0.05$, and $1.35 \pm 0.08 \mathrm{~mm}$ for the metallic spacers, and $1.25 \pm 0.08$ and $1.40 \pm 0.12 \mathrm{~mm}$ for the annular Teflon spacer.

Combinations of these parameters have given rise to patterns with a number of fingers varying from 8 to 80 . Producing patterns with less than eight fingers is nearly impossible in our conditions. It requires setting $R_{0}$ and $\Omega$ to values too small to avoid dripping of one oil in the other, as explained above.

Since there is no control of the setup temperature, and the viscosity of the liquids is highly sensitive to temperature variations (Figs. 1 and 2$)$, the viscosity contrast $A=\left(\mu_{2}\right.$ $\left.-\mu_{1}\right) /\left(\mu_{2}+\mu_{1}\right)$ has been slightly different from one experiment to another, within the interval $A=0.4-0.5$, following the variations in room temperature. It is known from numerical simulations that a strong sensitivity of the fingering dy-

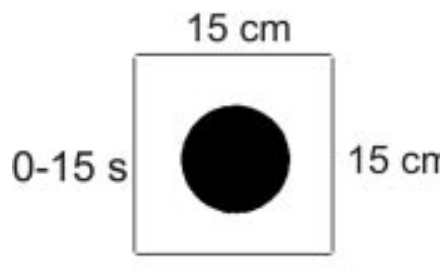

$41 \mathrm{~s}$

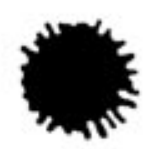

$63 \mathrm{~s}$
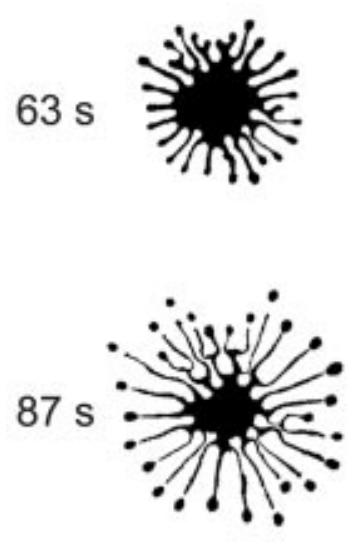

$31 \mathrm{~s}$
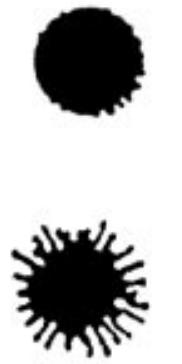

$51 \mathrm{~s}$
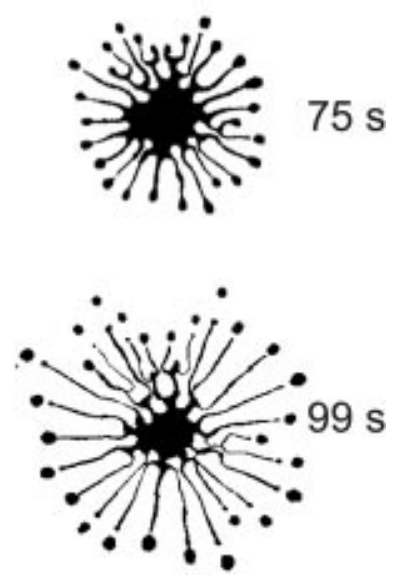

FIG. 3. Snapshots of the pattern formation process in an experiment with $b=1.4 \mathrm{~mm}, R_{0}=41 \mathrm{~mm}$, and $\Omega=210 \mathrm{rpm}$. The images are shown from left to right and from top to bottom.

namics and morphology to the viscosity contrast is observed at values of $A$ close to 1 , while no significant sensitivity to viscosity contrast is observed in the remaining interval of lower $A ., 5,6$

\section{Qualitative description of the patterns}

Once the initial condition has been prepared, the cell is set into motion. Figure 3 shows a temporal sequence of the patterns obtained in a typical experiment.

There is first a latency period $t_{l}$, dependent on the parameters of the experiment, in which the interface remains circular. After this period the interface develops small ripples, at the onset of instability. The pattern formed is not perfectly symmetric, due to unavoidable randomness in the initial condition, but it presents a regular arrangement of fingers of similar size.

The linear regime, in which the amplitude of the ripples is smaller than or comparable to their lateral size, is very short. Very quickly the interface perturbations grow in time, both inwards (vaseline displacing silicone) and outwards (silicone displacing vaseline), giving rise to a characteristic fingering pattern.

One of the most remarkable features of the temporal evolution of this pattern is the absence of competition between adjacent fingers of different sizes, in the sense that the number of growing fingers present in the linear regime is not modified during the development of the pattern. This is best 

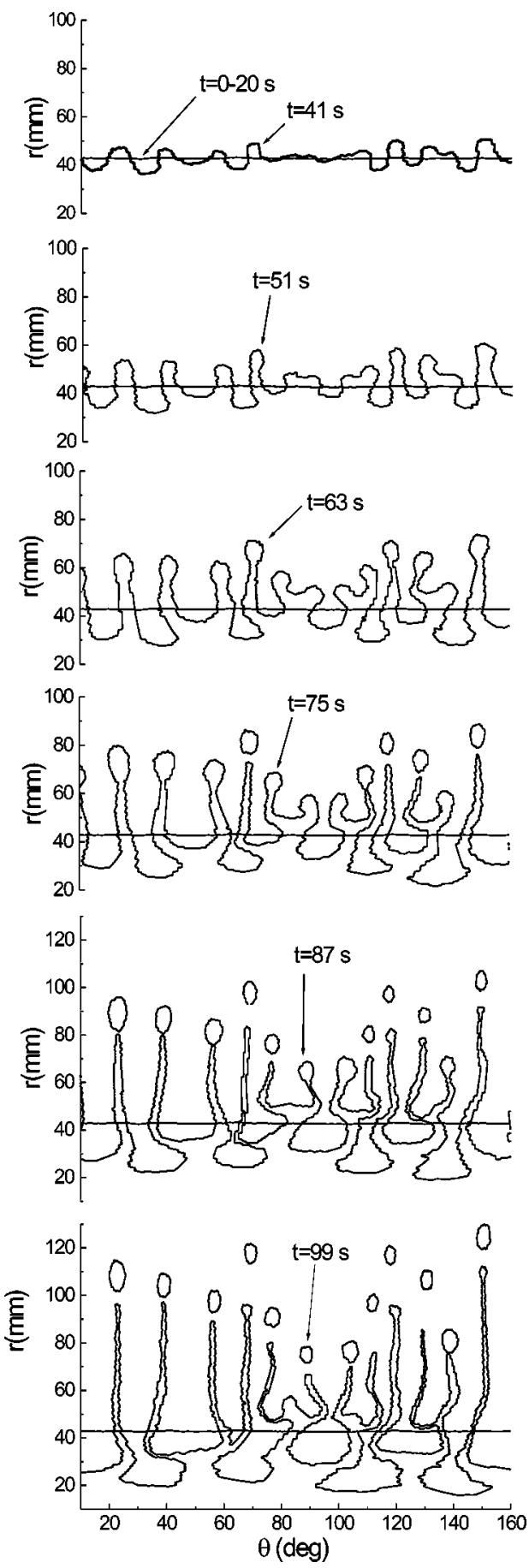

FIG. 4. Polar representation of the interfaces shown in Fig. 3. The initial condition is reproduced on each plot as a reference.

visualized in the plot of the interface radius as a function of the polar angle shown in Fig. 4. Represented in this way, the patterns show remarkable morphological similarities with the patterns obtained in the channel geometry, both numerically ${ }^{3}$ and experimentally, ${ }^{4}$ in the limit of low viscosity contrast (except for the breaking of up-down symmetry).

In our case, however, as the pattern goes into the deeply nonlinear regime, the fingers of silicone oil stretch and grow fast outwards, while the fingers of vaseline oil nearly stop as they approach the center of the cell. This asymmetric behavior is a purely geometrical effect not present in the channel

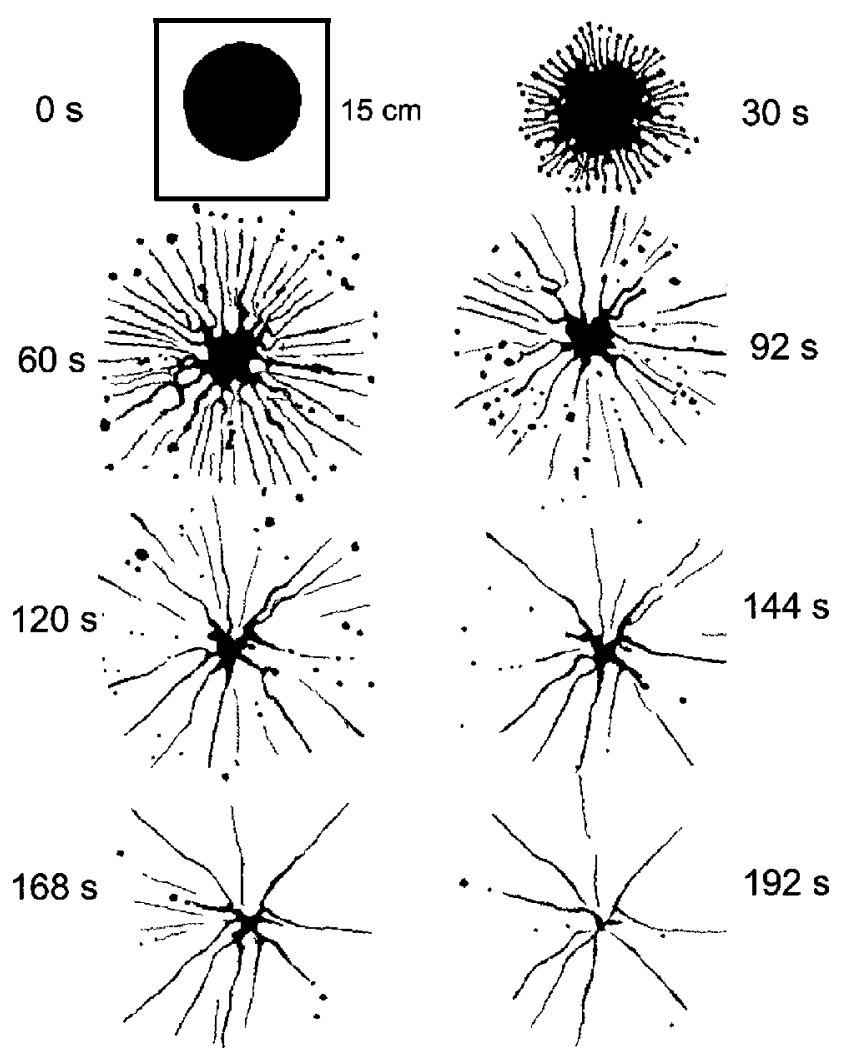

FIG. 5. Snapshots of the patterns obtained in an experiment with $b$ $=1.25 \mathrm{~mm}, R_{0}=54 \mathrm{~mm}$, and $\Omega=270 \mathrm{rpm}$, showing the latest stages of the pattern evolution. The images are shown from left to right and from top to bottom.

geometry. Figure 5 presents the sequence of patterns obtained in a second experiment, focusing on the nonlinear, latest stages of the dynamics.

A salient feature of our patterns is the strong tendency of the silicone fingers to pinch off, observed systematically in all experiments. Pinch-off follows the relatively fast stretching of the longer outgrowing fingers, which evolve into balloon-shaped fingers, with narrow filaments ending in a wider, rounded droplet. The pinch-off takes place at the point where the filament widens to form the droplet. A sequence of pictures illustrating this characteristic behavior is presented in Fig. 6. In some instances, provided that the filament of silicone oil receives enough flux from the cell center, a pinch-off event is followed by a series of new pinch-off events of the same finger, giving rise to a train of progressively smaller droplets, usually so small that can only be resolved once the cell is at rest.

After the pinch-off process, there is nearly no further displacement of the inner fluid outwards, and therefore no possibility for the vaseline fingers to grow inwards. When all fingers have pinched, the system must reorganize itself to continue the emission of the inner, denser liquid. We have observed two main mechanisms for this, both shown in Fig. 5: first, the reconnection of the incoming vaseline fingers, which results in a reduction of their number, and second the formation of new silicone fingers in the very late stages. These questions will be analyzed in detail in Sec. III F. Although the total amount of time for displacing most of the 


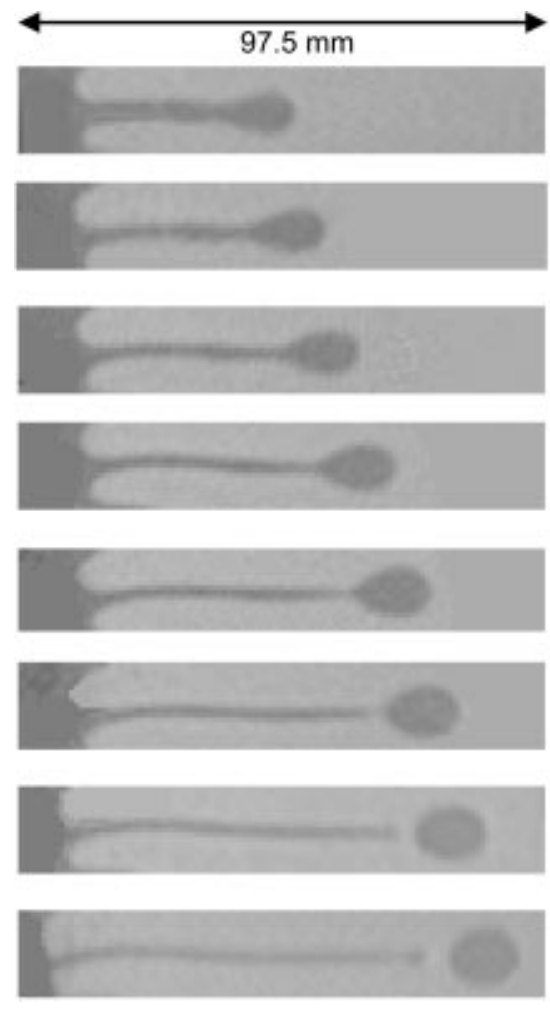

$109 \mathrm{~s}$

$115 \mathrm{~s}$

$121 \mathrm{~s}$

$127 \mathrm{~s}$

$133 \mathrm{~s}$

$139 \mathrm{~s}$

$146 s$

$154 \mathrm{~s}$

FIG. 6. Sequence of pictures extracted from an experiment with $b$ $=1.4 \mathrm{~mm}, R_{0}=37.5 \mathrm{~mm}$, and $\Omega=180 \mathrm{rpm}$.

mass initially present in the inner layer never exceeds $5 \mathrm{~min}$, it must be recognized that in the very late stages the pattern is probably also affected by the corrugation of the wetting layers coating the glass plates.

\section{ANALYSIS AND DISCUSSION}

\section{A. Hele-Shaw equations}

Navier-Stokes equation for the velocity field of a fluid, $\mathbf{V}$, observed in a reference frame rotating with angular frequency $\boldsymbol{\Omega}$, reads

$$
\begin{aligned}
\partial_{t} \mathbf{V}+(\mathbf{V} \cdot \nabla) \mathbf{V}= & -\frac{1}{\rho} \nabla p-\boldsymbol{\Omega} \times(\boldsymbol{\Omega} \times \mathbf{r}) \\
& -2 \boldsymbol{\Omega} \times \mathbf{V}+\nu \Delta \mathbf{V},
\end{aligned}
$$

where $\rho$ is the density of the fluid, $p$ is the hydrostatic pressure field, $\mathbf{r}$ is the position of the fluid element measured from the rotation axis, and $\nu$ is the kinematic viscosity, $\nu$ $=\mu / \rho$.

When this equation is particularized to a rotating HeleShaw cell with $\boldsymbol{\Omega}=\boldsymbol{\Omega} \hat{z}$, inertial terms are much smaller than viscous forces and pressure gradients, and the two terms on the left hand side are negligible. Considering a Poiseuille flow in the plane of the cell, and taking the average of the velocity field in the $z$ direction, we obtain ${ }^{8}$

$$
\mathbf{v}=-\frac{b^{2}}{12 \mu_{i}} \nabla\left(p_{i}-\frac{1}{2} \rho_{i} \Omega^{2} r^{2}\right)+\frac{b^{2}}{12 \mu_{i}} 2 \rho_{i} \Omega \hat{z} \times \mathbf{v},
$$

where $\mathbf{v}$ is the average velocity in the $r \phi$ plane.
The contribution proportional to $\Omega^{2}$ arises from centrifugal forces, and the one proportional to $\Omega$ arises from Coriolis forces. The role of these two contributions has been demonstrated in numerical simulations of large viscosity contrast flows. ${ }^{8}$ Centrifugal forces tend to stretch an initially circular droplet along the radial direction, producing elongated fingers which, due now to Coriolis forces, experiment a slight deviation from purely radial growth in the counter direction of cell rotation.

Coriolis effects, however, are negligible in our experiments. This is best seen by considering the reciprocal Eckman number introduced by Schwartz ${ }^{8}$

$$
\operatorname{Re} \equiv \frac{b^{2}}{12 \mu_{i}} \rho_{i} \Omega .
$$

The largest value that $\operatorname{Re}$ takes in our experiments is $\mathrm{Re}$ $\simeq 0.03$, which corresponds to the largest gap thickness used $b=1.4 \mathrm{~mm}$, the largest angular frequency accessible in our setup $\Omega=300 \mathrm{rpm}$, and the smallest kinematic viscosity $\mu / \rho=2 \times 10^{-4} \mathrm{~m}^{2} / \mathrm{s}$. Since $\operatorname{Re} \ll 1$, the Coriolis term is just a perturbation of the velocity of relative magnitude $2 \mathrm{Re}$, which can be safely neglected.

For typical experimental parameters $\mathrm{Re} \simeq 0.01$, and the typical radial velocity of well developed fingers is $U_{r}$ $\simeq 1 \mathrm{~mm} / \mathrm{s}$. Under these conditions, Coriolis forces would produce tangential velocities $U_{\phi} \simeq 0.02 \mathrm{~mm} / \mathrm{s}$, which cannot be resolved in the present experiments. For Coriolis effects to be observable in our experimental setup, the kinematic viscosity of the fluids should be smaller by about 2 orders of magnitude. Water, for instance, would show chiral growth associated with Coriolis forces even for narrow gaps and low angular velocities.

Neglecting the Coriolis term in Eq. (2), Darcy's law can be written as

$$
\mathbf{v}_{i}=\boldsymbol{\nabla} \phi_{i}, \quad i=1,2
$$

with

$$
\phi_{i}=-\frac{b^{2}}{12 \mu_{i}}\left(p_{i}-\frac{1}{2} \rho_{i} \Omega^{2} r^{2}\right), \quad i=1,2 .
$$

The condition of incompressibility of the two liquids, $\boldsymbol{\nabla} \cdot \mathbf{v}=0$, leads to the Laplace equation for the velocity potential in the bulk

$$
\Delta \phi_{i}=0 .
$$

To complete the set of equations for the free boundary problem in two dimensions, we must provide kinematic and dynamic boundary conditions at the interface. The two boundary conditions considered usually are, first, the continuity of the normal velocity at the interface

$$
v_{n}=\hat{n} \cdot \boldsymbol{\nabla} \phi_{1}=\hat{n} \cdot \boldsymbol{\nabla} \phi_{2}
$$

and, second, a linear relation between the local pressure jump at the interface and its curvature

$$
p_{2}-p_{1}=\sigma \kappa,
$$

where $\sigma$ is the interfacial tension between the two liquids and $\kappa$ is the local curvature of the interface (considered a one-dimensional line). 
Although it has been often ignored in the literature, this last equation is not strictly correct, particularly in the circular geometry. ${ }^{14}$ Equation (8) actually comes from the quasistatic condition of local mechanical equilibrium:

$$
\hat{n} \cdot\left[\overrightarrow{\boldsymbol{\tau}}_{1}-\overrightarrow{\boldsymbol{\tau}}_{2}\right] \cdot \hat{n}=\sigma \kappa,
$$

where $\overrightarrow{\boldsymbol{\tau}}$ is the local stress tensor. The effect of the normal velocity gradients may be negligible in most situations in the channel geometry, in particular in the linear regime, since the normal velocity gradients give rise to higher order contributions. Then the above condition reduces indeed to the usual one Eq. (8). However, in a radial cell, normal velocity gradients on a circular interface do not vanish and the condition leads to

$$
p_{2}-p_{1}-2\left(\mu_{2} \partial_{r} v_{r, 2}-\mu_{1} \partial_{r} v_{r, 1}\right)=\sigma \kappa,
$$

where $v_{r, 1}$ and $v_{r, 2}$ are the radial components of the velocity field in each of the two fluids. The additional terms in Eq. (10) will turn out to be relevant for our experiments, as shown later.

The boundary conditions specified above are a simplification of the real three-dimensional problem. In practice the interface is a two-dimensional object with two local main curvatures, $\kappa_{\|}$and $\kappa_{\perp}$, in the directions parallel and perpendicular to the plane of the cell respectively. This gives rise to a number of complications:

(i) Depending on the wetting properties of the fluids and their relative velocity, the interface may either leave behind or absorb a liquid film on each glass plate. This modifies the balance of mass at the interface and, consequently, the kinematic boundary condition (7).

(ii) The thickness of the wetting films, and the dynamic contact angle at the contact line of the interface with the glass plates, may be sensitively dependent on the interface velocity. As a result, the curvature $\kappa_{\perp}$ is not constant [as implicitly assumed in Eq. (8)], but a function of the local interface velocity. This affects the dynamic boundary condition (8).

These effects have been studied in detail in the channel geometry, for air displacing a wetting liquid (viscosity contrast $A=1$ ). Park and Homsy ${ }^{15}$ proved that Eq. (8) should read

$$
p_{2}-p_{1}=\sigma\left[\frac{2}{b}+\frac{\pi}{4} \kappa_{\|}\right]
$$

for $\mathrm{Ca} \ll 1$, where $\mathrm{Ca}$ is the capillary number $\mu V / \sigma$, with $V$ the velocity of the interface. The next additional correction, derived by Schwartz, ${ }^{16}$ takes into account the influence of the viscous wetting film left on the plates as the interface moves

$$
p_{2}-p_{1}=\sigma\left[\frac{2}{b}+\frac{\pi}{4} \kappa_{\|}\right]+\frac{2 \sigma J}{b} \mathrm{Ca}^{2 / 3},
$$

where $J \simeq 3.8$ in the formulation of Park and Homsy.

A similar theoretical effort (including the extra stresses from the velocity normal gradients) has not been carried out for the radial geometry. To our knowledge, Maxworthy has been the only author to consider kinetic corrections to the boundary conditions in experiments on this geometry, by as- suming that the expressions valid in the channel geometry could be directly translated to the radial geometry. ${ }^{17}$ In our specific case, however, $Q=0$ and therefore the interface is at rest (in the reference frame rotating with the cell) at the onset of the instability. Any corrections proportional to power laws of the interface velocity will be correspondingly small. Thus, it is reasonable to assume that the dominant correction to the two-dimensional boundary conditions, in the linear regime, will come only from the influence of the wetting films on the curvature $\kappa_{\perp}$ of the meniscus. Following the derivation of Park and Homsy for the channel geometry, ${ }^{15} \mathrm{Eq}$. (11), the correction is simply a factor $\pi / 4$ multiplying the surface tension. Assuming that a similar correction would apply in our case, but eventually with a different numerical factor, we conclude that a convenient way to take three-dimensional effects into account, in the two-dimensional boundary conditions of our problem, is to consider $\sigma$ in Eq. (8) as an effective interfacial tension, and leave it as a free parameter to be determined a posteriori. This is even more reasonable if we recall that the actual value of $\sigma$ is itself subject to a large experimental uncertainty, as discussed in the previous section.

\section{B. Linear stability analysis}

First we make use of Eq. (8) as a dynamic boundary condition. The linear dispersion relation of an initially circular interface reads in this case ${ }^{9}$

$$
\omega(n)=\widetilde{\Omega} n-\frac{\widetilde{\sigma}}{R_{0}^{3}} n\left(n^{2}-1\right),
$$

where $\omega(n)$ is the growth rate of an infinitesimal perturbation of the circular interface of the form $\delta e^{i n \theta}$ (mode $n$ ), and

$$
\widetilde{\Omega}=\frac{\Omega^{2} b^{2}}{12} \frac{\rho_{2}-\rho_{1}}{\mu_{2}+\mu_{1}}, \quad \widetilde{\sigma}=\frac{b^{2}}{12} \frac{\sigma}{\mu_{2}+\mu_{1}} .
$$

In our case $\rho_{2}>\rho_{1}$, making the centrifugal force destabilizing for all modes, as expected. The interfacial tension does not affect the stability of the mode $n=1$ (which preserves the circular shape) but stabilizes all modes $n>1$. Finally, since $Q=0$, the viscosity contrast does not play any role at the linear level.

The fastest growing mode $n_{m}$ and the marginal mode $n_{c}$ (separating unstable from stable modes) are given by

$$
n_{m}=\sqrt{\frac{1}{3}(1+S)}, \quad n_{c}=\sqrt{1+S} .
$$

Both depend on the parameter $S=R_{0}^{3} \widetilde{\Omega} / \widetilde{\sigma}$, the ratio of centrifugal to capillary forces, which plays the role of an inverse dimensionless surface tension. Since $\widetilde{\sigma}$ is very small in our experiments, we have $S \gg 1$ in most experimental conditions. Then, $n_{m}$ and $n_{c}$ can be approximated by

$$
n_{m} \simeq \sqrt{\frac{S}{3}}, \quad n_{c} \simeq \sqrt{S} .
$$

It is not difficult to show that, in this same limit, the growth rate of the fastest growing mode is given by

$$
\omega\left(n_{m}\right)=\frac{2}{3} n_{m} \omega(1) .
$$


When instead of Eq. (8) we consider Eq. (10) as the proper boundary condition the linear dispersion relation changes to

$$
\begin{aligned}
\omega^{*}(n) & =\frac{1}{1+\frac{1}{6} n(n-A)\left(\frac{b}{R_{0}}\right)^{2}}\left[\widetilde{\Omega} n-\frac{\widetilde{\sigma}}{R_{0}^{3}} n\left(n^{2}-1\right)\right] \\
& \simeq \frac{1}{1+\frac{1}{6} n^{2} \epsilon^{2}}\left[\widetilde{\Omega} n-\frac{\widetilde{\sigma}}{R_{0}^{3}} n^{3}\right]
\end{aligned}
$$

where $\epsilon=b / R_{0}$ and the approximation is valid when $n \gg 1$. This dispersion relation differs from the previous one in the prefactor, that introduces a dependence on the gap spacing $b$. The correction is important only when $n \epsilon \sim 1$, i.e., for modes of large wave number $n \sim R_{0} / b$. While $n_{c}$ is not modified, the wave number of the fastest growing mode is now given by

$$
\begin{aligned}
n_{m}^{*} & =\sqrt{\frac{1}{2}\left[\sqrt{\left(S+\frac{18}{\epsilon^{2}}\right)^{2}+S \frac{24}{\epsilon^{2}}}-\left(S+\frac{18}{\epsilon^{2}}\right)\right]} \\
& =\sqrt{\frac{S}{3}\left[1+\left(\frac{\epsilon^{2}}{9}\right) \frac{S}{3}+O\left(\epsilon^{4} S^{2}\right)\right] .}
\end{aligned}
$$

The number $n_{m}^{*}$ coincides with $n_{m}$ except for a correction depending on the dimensionless parameter $\epsilon^{2} S$. This correction results in larger wave numbers for larger gap spacings.

The low value of the interfacial tension in our experiments gives rise to a large number of fingers and makes the correction above non-negligible. On the other hand but for the same reason $n \gg A$, making the correction independent of the viscosity contrast.

\section{Number of fingers emerging from the linear instability}

Since the growth of the unstable modes in the linear regime is exponential, it is reasonable to expect that the fastest growing mode (as given by the maximum of the linear dispersion relation) determines the typical number of fingers formed at the onset of instability, provided that the noise is sufficiently weak and uniformly distributed in all modes. In experiments, however, this is not quite the case and, since the bandwidth of unstable modes is large and the duration of the linear regime is very short, the direct experimental verification of the linear dispersion relation is rather difficult. ${ }^{9,17,18}$ In the high- $A$ limit, in addition, the nonlinear regime presents a dynamic competition between neighbor fingers of differing sizes, which results in a systematic reduction of the number of fingers present in the nonlinear regime.

Low- $A$ flows, however, exhibit no competition between neighbor fingers, not only in the linear and weakly nonlinear regime but in the deeply nonlinear regime as well. This guarantees that the wave number of the mode of largest amplitude at the end of the linear regime coincides with the number of fingers observed in a given experiment. In summary,

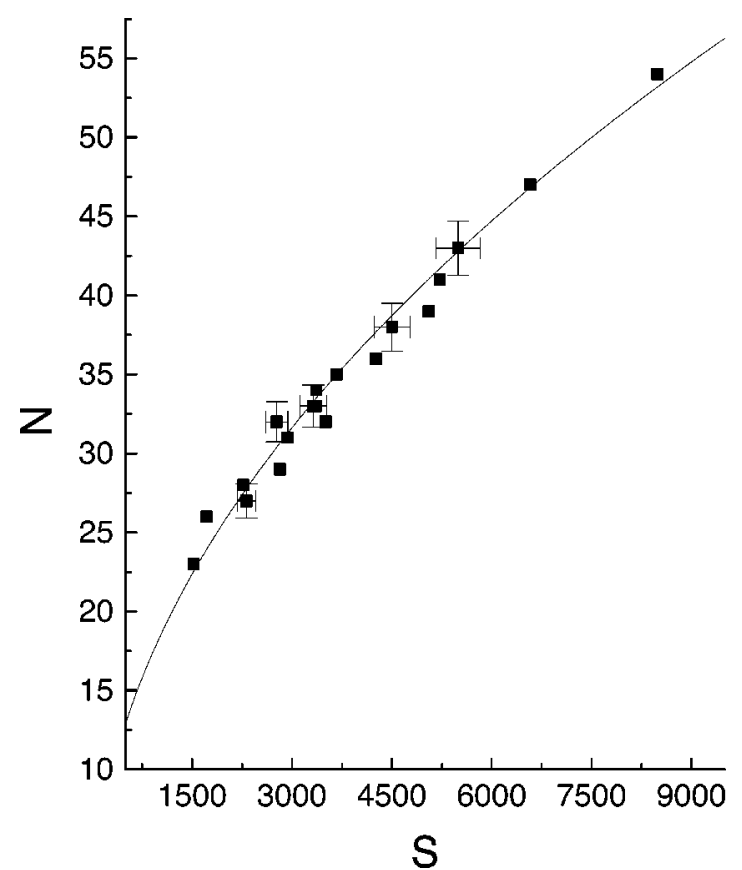

FIG. 7. (Filled squares) Number of fingers measured in a series of experiments with gap spacing $b=1.4 \mathrm{~mm}$ and different values of $R_{0}$ and $\Omega$, as a function of the dimensionless parameter $S$. (Solid line) Least-squares fit of the data to a function $n_{m} \simeq \sqrt{S / 3}$, giving an effective interfacial tension $\sigma$ $=1.9 \mathrm{mN} / \mathrm{m}$

the lack of finger competition makes low- $A$ flows particularly suitable to check the predictions of a linear stability analysis.

Figure 7 presents the number of fingers measured in experiments with $b=1.4 \mathrm{~mm}$ and different values of $R_{0}$ and $\Omega$ (allowing for the control parameter $S$ to vary between 1500 and 9000), as a function of $S$. In each experiment the number of fingers has been determined at the beginning of the nonlinear regime and, also, at the end of the nonlinear regime when the first droplets reach the cell edge. Both countings never differ by more than $5 \%$. The difference is represented by the vertical error bars. The error bars for $S$, on the other hand, reflect the experimental uncertainties in $\rho_{1}, \rho_{2}, R_{0}$, and $\Omega$, but not in the interfacial tension $\sigma$. The solid line running through the experimental points is a fit of the form $n_{m} \simeq \sqrt{S / 3}$, Eq. (16), in which $\sigma$ has been left as a fitting parameter. The fit gives $\sigma=1.9 \pm 0.1 \mathrm{mN} / \mathrm{m}$.

Compared to previous analysis of the linear dispersion relation, ${ }^{9,17,18}$ our experimental data show a remarkably small dispersion. This reflects the good reproducibility of the experiments (the number of fingers formed in identical experimental conditions is reproducible within less than 5\%), thanks to the absence of finger competition.

We must emphasize that the interfacial tension obtained in the fit, although consistent with our experimental estimation of this parameter, does not necessarily coincide with the actual value of $\sigma$. First, we recall that wetting effects renormalize $\sigma$ by an unknown factor (the factor $\pi / 4$ in channel geometry and $A=1$ ), and second, it remains to be determined whether the gap spacing $b$ has an influence on the effective value of $\sigma$, as predicted by Eq. (19). 


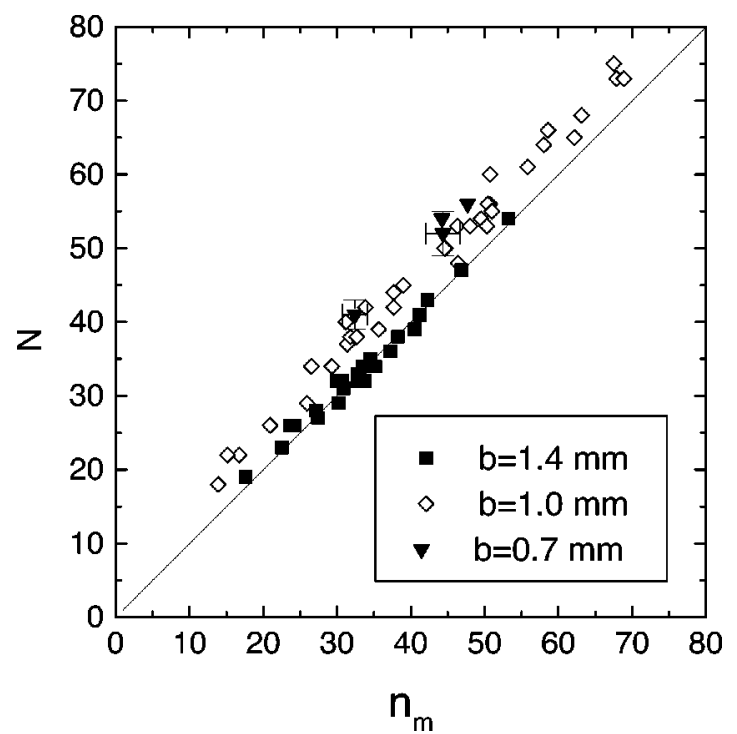

FIG. 8. (Symbols) Number of fingers, $N$, measured in experiments with different values of $R_{0}, \Omega$, and three gap spacings $b$, against the number of fingers predicted by the fastest growing mode of the linear dispersion relation, $n_{m} \simeq \sqrt{S / 3}$. The straight line reproduces the fit with $\sigma=1.9 \mathrm{mN} / \mathrm{m}$ shown in Fig. 7.

We have addressed this second question by performing similar experiments with different gap spacings $(b=1.4,1.0$, and $0.7 \mathrm{~mm}$ ). The results are shown in Fig. 8, in which the number of fingers observed experimentally, $N$, is represented against the prediction for $n_{m}$ given by Eq. (16). The solid squares are the data for $b=1.4 \mathrm{~mm}$ presented already in Fig. 7 , and the straight line the corresponding fit with $\sigma$ $=1.9 \mathrm{mN} / \mathrm{m}$. It is clear from the figure that the other data points are shifted systematically from the linear fit. The error bars (shown only for two points for clarity) reveal that the deviation from the fit is not accidental. We conclude that a variation of the gap spacing seems to change the effective interfacial tension in a systematic way.

This result can be accounted for by the modified dynamic boundary condition (10) discussed in Sec. III A. This is demonstrated in Fig. 9, where the same data points are represented against $n_{m}^{*}$, given by Eq. (19), and are seen to collapse in a single straight line corresponding to an effective interfacial tension $\sigma=1.4 \mathrm{mN} / \mathrm{m}$. It becomes clear that working with two similar liquids makes the interfacial tension very small, which favors a large number of fingers, so that the small correction that leads to $n_{m}^{*}$ is relevant in our experiments.

This analysis has provided us with an accurate value of the effective interfacial tension, $\sigma=1.4 \mathrm{mN} / \mathrm{m}$. In the case where we had a precise experimental determination of the actual interfacial tension, we would be able to determine the correction factor due to wetting effects. Currently we can only mention that if we use the value measured by the spinning drop method, $\sigma=1.8 \mathrm{mN} / \mathrm{m}$, which is subject to a large uncertainty, we get a correction factor very close to $\pi / 4$.

\section{Empirical scaling of the patterns}

An analysis ${ }^{7}$ of the Hele-Shaw equations presented in Sec. III A shows that the experiments are controlled essen-

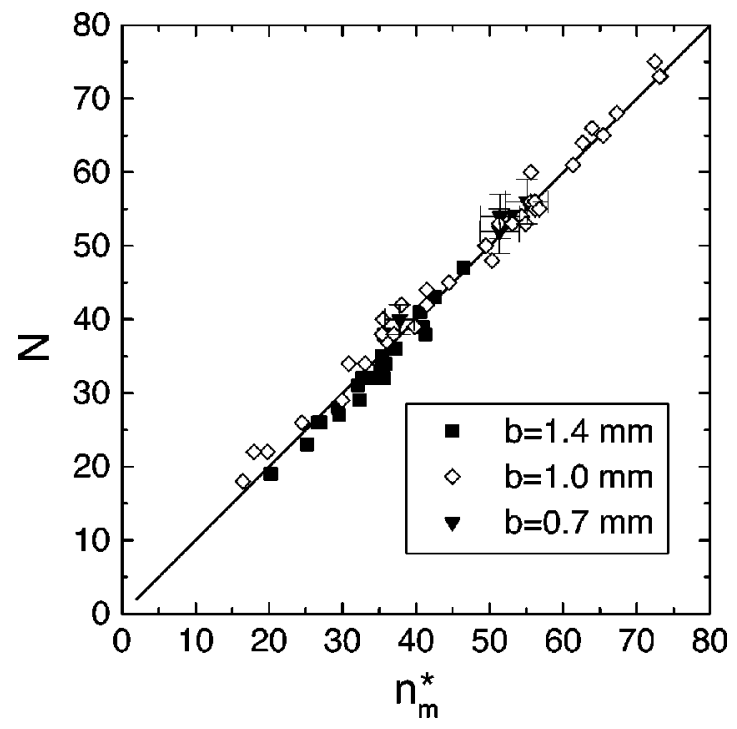

FIG. 9. Data collapse of the measured number of fingers, $N$, achieved by plotting this number against $n_{m}^{*}$ [the number of fingers predicted by Eq. (19)] with an effective surface tension $\sigma=1.4 \mathrm{mN} / \mathrm{m}$.

tially by two dimensionless parameters, the viscosity contrast $A$ and the ratio of centrifugal to capillary forces $S$. The first of them (not varied in the present experiments) has no influence on the linear regime, provided that $Q=0$, but plays an important dynamic role in the nonlinear regime by controlling finger competition. The second one determines the dominant wave number emerging from the linear regime, which remains dominant throughout the whole evolution for low $A$-flows. On the other hand, our discussion about the appropriate dynamic boundary condition in the circular geometry has shown that the gap spacing also plays a measurable role in the linear regime and, thus, introduces a third dimensionless group $\epsilon$. The influence of this third parameter in the deeply nonlinear regime is more difficult to assess but we do not expect it to be very significant.

The linear dispersion relation suggests a simple way to make length and time dimensionless, using $R_{0}$ (initial radius) and $1 / \omega(1)$ [where $\omega(1)$, the growth rate of the mode $n$ $=1$, is simply $\widetilde{\Omega}]$, as the characteristic length and time scales.

In our experiments of high viscosity contrast flows ${ }^{9,14}$ this simple choice proved adequate to scale several measures of the patterns, including the latency period before the development of the pattern, the radius of gyration of the pattern, the radial extent of the mixing zone, and others. This choice, however, did not provide a good scaling of the interface stretching. This lack of scaling was attributed to the sensitivity of interface stretching to capillary forces.

A natural way to introduce a dimensionless time scale that takes into account the stabilizing role of the in-plane surface tension $\sigma$ is to replace $t \cdot \omega(1)$ by $t \cdot \omega\left(n_{m}\right)$. Since, according to Eq. (17), $\omega\left(n_{m}\right) \sim \omega(1) \cdot n_{m}, \quad$ and $n_{m}$ $\simeq(S / 3)^{1 / 2}$, this new time scale introduces $S$ (the ratio of centrifugal to capillary forces) in the empirical scaling.

On the other hand, our study of the linear regime (Sec. III C) has showed that replacing $n_{m}$ by $n_{m}^{*}$ captures the slight 


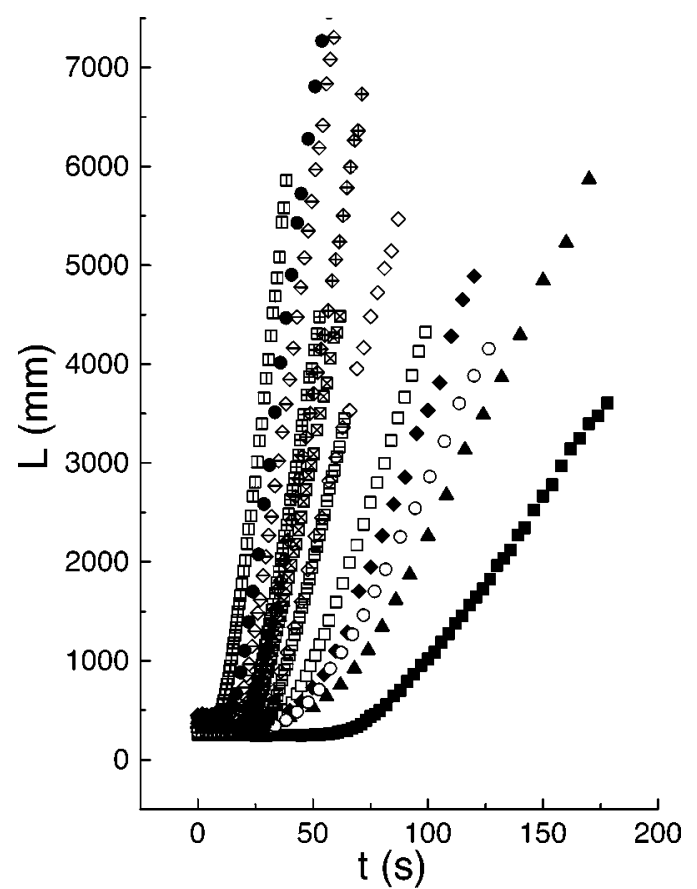

FIG. 10. Interface stretching as a function of time, in a series of experiments with different $b, R_{0}$, and $\Omega$.

dependence on $\epsilon$ introduced by the modified boundary condition (10). A suitable dimensionless time scale is therefore given by $t \cdot \omega(1) \cdot n_{m}^{*}$.

In order to make a quantitative characterization of the spatiotemporal evolution of the morphological instability, we study in some detail the interface stretching, the latency time before the onset of instability, the radius of gyration of the pattern, and the mass of the droplets emitted by the radially outgrowing fingers.

\section{Latency period}

The latency period, $t_{l}$, is the time interval in which the circular oil bubble (initial condition) remains circular, within experimental resolution, before the instability sets in and the pattern develops appreciably.

All measures of the pattern (such as the interface stretching, the radius of gyration, etc.), when plotted as a function of time, remain constant during $t_{l}$, and then increase sharply as the instability develops and the pattern enters the nonlinear regime.

In order to characterize $t_{l}$, we consider in particular the time evolution of the interface length in experiments with different gap thickness, initial radius, and rotation frequency, reported in Fig. 10. The interface length $L$ remains at its initial value $2 \pi R_{0}$ for a period of time, increases sharply in the linear regime, and then grows at a nearly constant rate in the nonlinear regime. Our data points follow the interface stretching until a first point of the interface reaches the cell edge. $t_{l}$ is obtained by a backward extrapolation of this last regime, thus measuring the time at which the instability would have set in, had the interface length always grown at the same rate.

Figure 11 presents the results of the latency time deter-

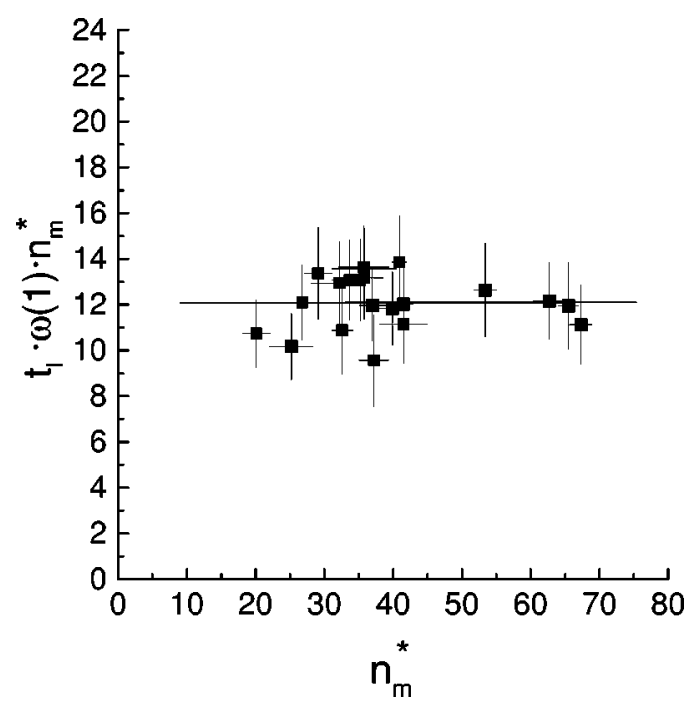

FIG. 11. Dimensionless latency time, $t_{l} \cdot \omega(1) \cdot n_{m}^{*}$, as a function of $n_{m}^{*}$, in a series of experiments with different $b, R_{0}$, and $\Omega$.

mined in this way, in the dimensionless form $t_{l} \cdot \omega(1) \cdot n_{m}^{*}$ versus the number of fingers formed in the experiment, represented by $n_{m}^{*}$. We recall that $n_{m}^{*}$ depends on experimental parameters through the two dimensionless groups $S, \epsilon$. The data show that, within the large experimental uncertainties in the determination of the latency time, $t_{l} \cdot \omega(1) \cdot n_{m}^{*}$ is practically a constant (12 \pm 2$)$ for all the experiments considered. This observation is consistent with a scenario in which the noise present in the initial condition has similar amplitude for all possible modes and is sufficiently weak. This ensures that the fastest growing mode, whose growth rate is proportional to $\omega(1) \cdot n_{m}^{*}$, has a much larger amplitude than any other mode at the end of the linear regime, and is therefore the first one to show up in the pattern.

A collapse of the latency time was also obtained for high viscosity contrast flows, but there $t_{l} \cdot \omega(1)$ rather than $t_{l}$ $\cdot \omega(1) \cdot n_{m}^{*}$ was independent of the number of fingers. ${ }^{9}$ The reason for the different behavior must be found in the different wetting conditions at the interface, since high viscosity contrast experiments were carried out in a dry (not prewetted) cell.

\section{Interface stretching}

The pronounced stretching of the interface presented in Fig. 10 is represented in dimensionless variables, $L / R_{0}$ versus $t \cdot \omega(1)$, in Fig. 12. These variables do not produce a collapse of the different curves. Actually, the mean slope of the different curves in the sharp growth region can be seen to depend linearly on the number of fingers, $N$, as shown in Fig. 13. The linear fit provides

$$
V_{s} \equiv \frac{1}{R_{0} \omega(1)} \frac{d L}{d t}=60+3.2 N .
$$

The interpretation of this result is that the interface stretching rate $\left(V_{s}\right)$ follows two different time scales simultaneously. The independent term, 60, reflects a scaling of $t$ with $\omega(1)$, which is dominant for a small number of fingers-infrequent 


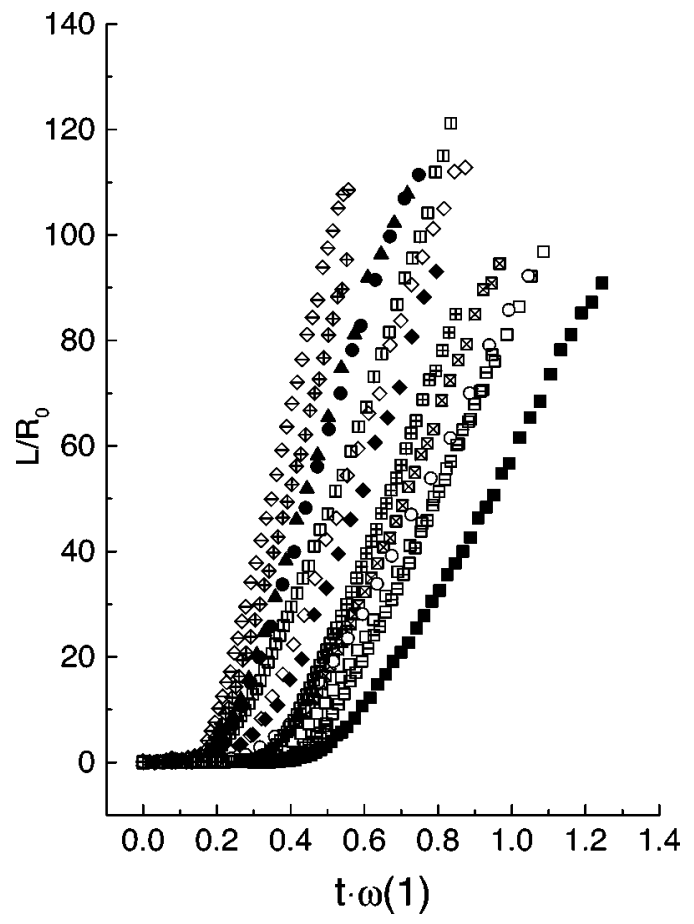

FIG. 12. Dimensionless interface stretching, $L / R_{0}$, as a function of dimensionless time, $t \cdot \omega(1)$, for the experiments represented in Fig. 10.

in our experiments. The term proportional to $N$ reflects a scaling of $t$ with $\omega(1) \cdot n_{m}^{*}$, which is dominant for a large number of fingers.

The linear dependence on $N$ in Eq. (20) corresponds to a scenario in which: (i) all fingers grow simultaneously at roughly the same speed and (ii) in the highly nonlinear re-

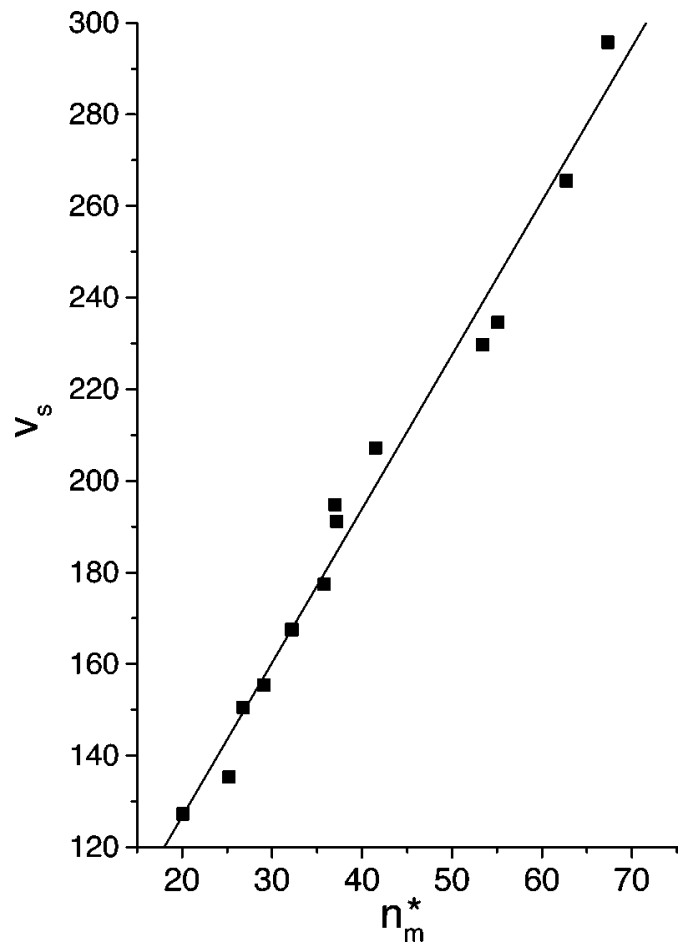

FIG. 13. Dimensionless stretching rate, $V_{s}$, vs number of fingers, $N$. The solid line is a least-squares linear fit of the data.
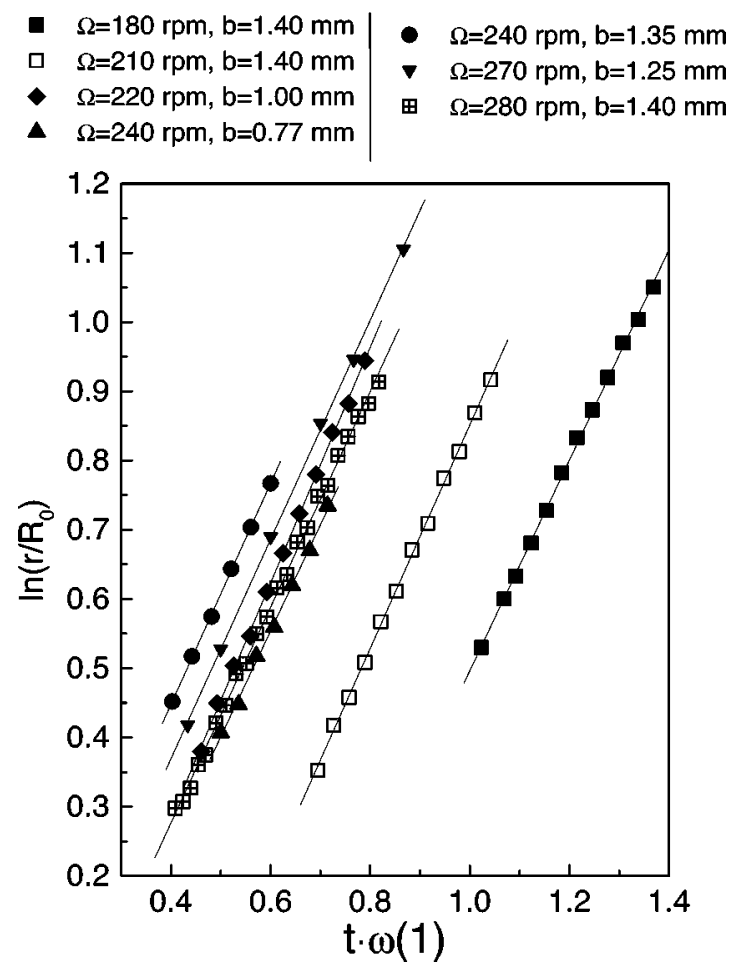

FIG. 14. Plot of $\ln \left(r / R_{0}\right)$ as a function of dimensionless time $t \cdot \omega(1)$. The radial coordinate $r$ gives the distance of the silicone oil droplets to the rotation axis. The average slope of the linear fits is $1.6 \pm 0.1$.

gime the contribution of a finger to the overall interface length $L$ comes basically from its two lateral, nearly parallel walls; the contribution, therefore, is twice its radial size, measured from the beginning of the finger to the end of the droplet. To verify this picture, we have measured directly the growth of the fingertips (radius $r$ ) in a large number of experiments. The experimental results (Fig. 14) show that $\ln \left(r / R_{0}\right)$ grows linearly with $\left(t-t_{0}\right) \cdot \omega(1)$ (where $t_{0}$ is the time when the finger is formed), and the slope is practically independent of experimental parameters: $\alpha=1.60 \pm 0.15$. Consequently, if we consider that the dominant contribution to the interface stretching is the stretching of the finger, which is particularly accurate when the number of fingers is large enough, we have $L \simeq 2 N r \simeq 2 N R_{0} \exp \left[\alpha\left(t-t_{0}\right) \omega(1)\right]$ $\simeq 2 N R_{0}\left[1+\alpha\left(t-t_{0}\right) \omega(1)\right]$, and thus

$$
\frac{1}{R_{0} \omega(1)} \frac{d L}{d t} \simeq 2 \alpha n_{m}^{*} \simeq 3.2 N
$$

in excellent agreement with the result of Eq. (20) derived directly from the experiments for large $N$.

From the former discussion it follows that, for large $N$, the interface length should grow with a time scale $t \cdot \omega(1)$ $\cdot n_{m}^{*}$. The corresponding data collapse is shown in Fig. 15 .

The simple model of the evolution of the pattern outlined in this section will be validated in forthcoming sections through other characterizations of the spatio-temporal dynamics. 


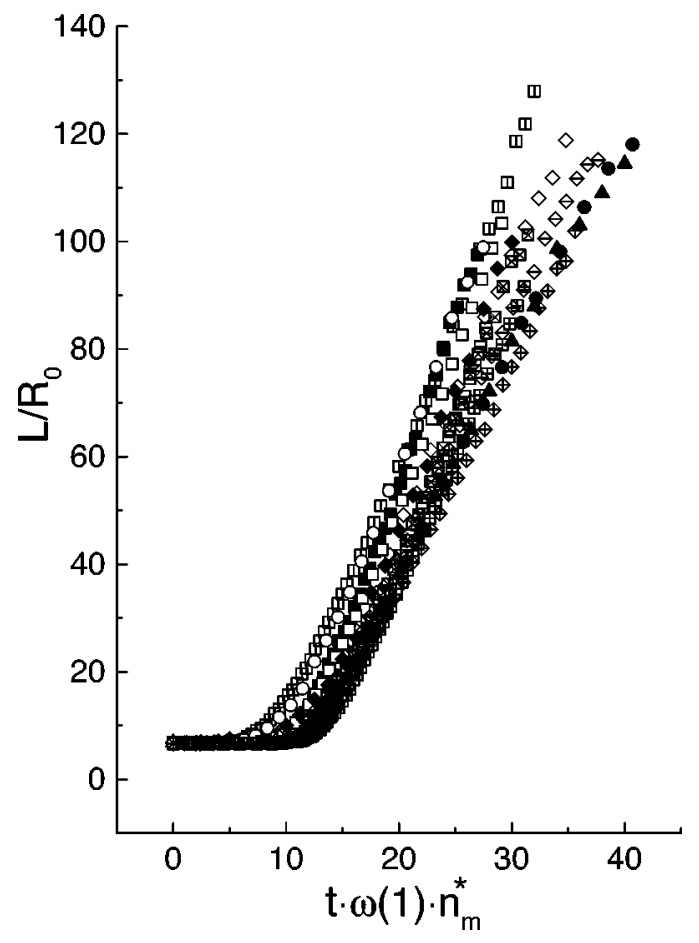

FIG. 15. Dimensionless interface stretching, $L / R_{0}$, as a function of dimensionless time, $t \cdot \omega(1) \cdot n_{m}^{*}$, for the experiments represented in Fig. 10.

\section{Mass distribution}

A different characterization of the morphological instability, complementary of the interface stretching, is provided by its radial mass distribution. "Mass" refers here to the two-dimensional area covered by the pattern. The properties of this distribution are measured by the moments $R_{n}$, defined as

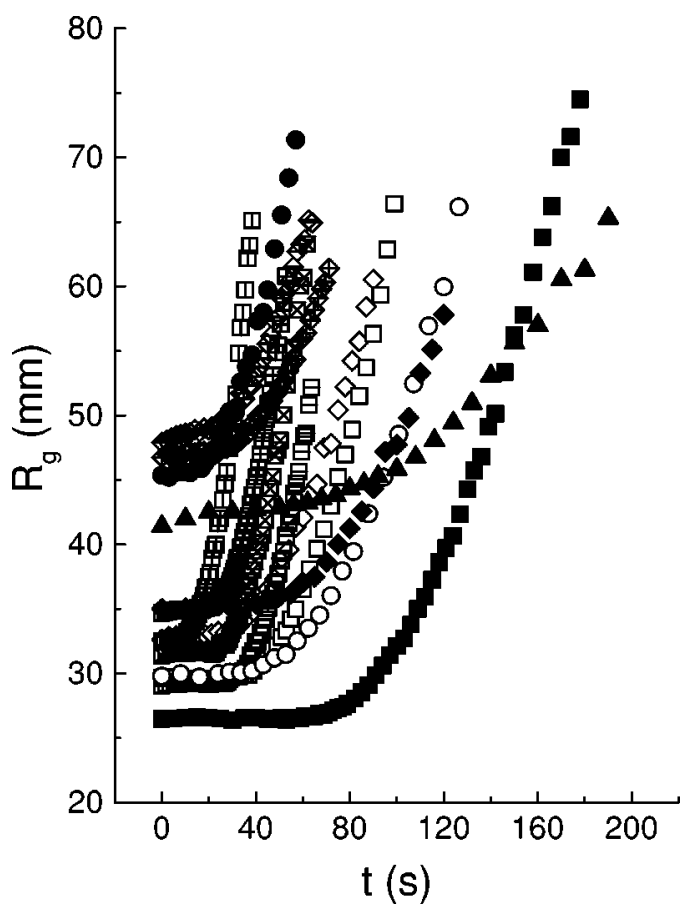

FIG. 16. Radius of gyration of the patterns as a function of time.

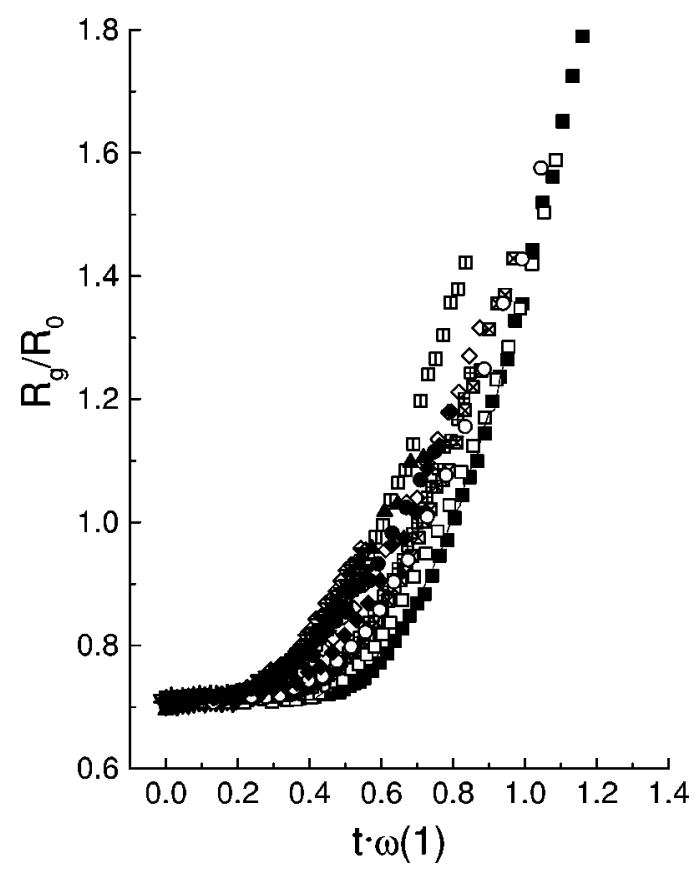

FIG. 17. Dimensionless radius of gyration, $R_{g} / R_{0}$, as a function of dimensionless time, $t \cdot \omega(1)$.

$$
R_{n}=\left[\frac{\int_{0}^{2 \pi} d \phi \int_{0}^{r(\phi)} r^{n} r d r}{\int_{0}^{2 \pi} d \phi \int_{0}^{r(\phi)} r d r}\right]^{1 / n} \quad n=1,2, \ldots
$$

The first moment is the average radius of the pattern. The second moment of the distribution is the so called "radius of gyration" $R_{g}$.

The time evolution of $R_{g}$ for our set of experiments is presented in Fig. 16. The overall picture shows a radius of gyration that remains constant during the latency time, starts growing in the linear regime, and increases exponentially in the nonlinear regime, until the droplets at the fingertips reach the end of the cell and no more data are available.

We have verified that the proper time scale to collapse the growth of the dimensionless radius of gyration, $R_{g} / R_{0}$, is $t \cdot \omega(1)$. The collapse is shown in Fig. 17. Not surprisingly, the different curves are laterally displaced, since the time scale of the latency time is $t \cdot \omega(1) \cdot n_{m}^{*}$.

The result presented in Fig. 17 can be understood in the same scenario described above: all fingers grow simultaneously, at a similar rate that scales with $t \cdot \omega(1)$. Making the assumption that the main contribution to the growth of $R_{g}$ comes from the droplet-shaped terminations of the fingers, and taking into account that there are $n_{m}^{*}$ fingers, we conclude that the mass carried by each finger and droplet must be inversely proportional to the number of fingers, for $R_{g} / R_{0}$ to scale with $t \cdot \omega(1)$ rather than with $t \cdot \omega(1) \cdot n_{m}^{*}$.

This conclusion is consistent with the morphology of the patterns (Fig. 3), where we see that, in the mixing zone, the pattern is formed by an alternancy of outward-growing silicone fingers and inward-growing vaseline fingers, all of similar width. Thus, the silicone fingers take up nearly the same room in all the experiments, independently of the number of growing fingers, $n_{m}^{*}$, each finger carrying an amount of fluid proportional to $1 / n_{m}^{*}$. 


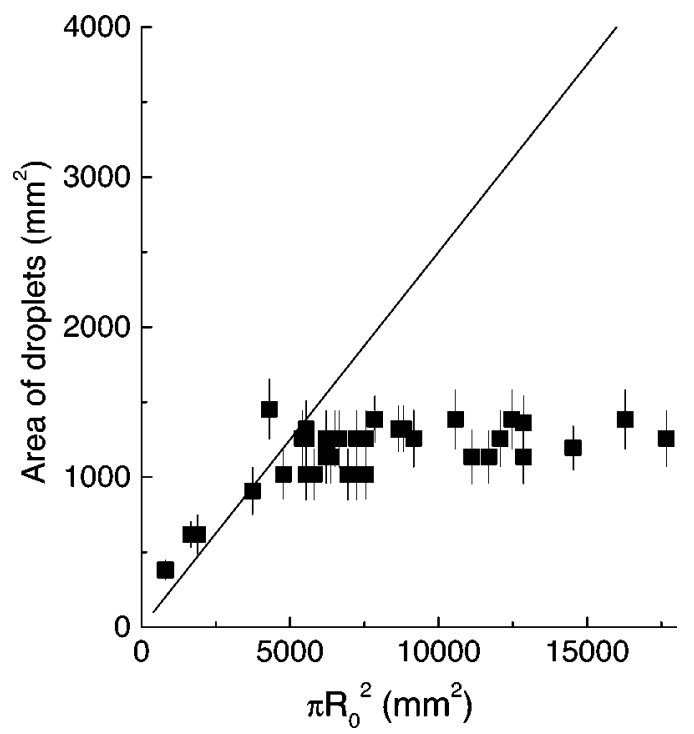

FIG. 18. Total area covered by the silicone oil droplets, after the first pinchoff process, vs the area covered by the initial condition. The solid line shows the behavior expected if the two magnitudes were mutually proportional.

Another consequence of this picture is that the total amount of silicone oil displaced outwards (in the outgrowing fingers) will be a constant, irrespective of the initial radius $R_{0}$. Only at small enough $R_{0}$, when the overall amount of silicone oil is too small to provide for this constant value, should a cutoff to this behavior be expected. The implications of this result on the size and overall mass of the droplets formed by finger pinchoff are discussed in the next section.

\section{E. Mass of emitted drops}

A typical sequence of the pinch-off process that gives rise to the release of a silicone oil droplet is shown in Fig. 6. We observe that the droplet at the fingertip widens as the finger stretches into an elongated filament. This continues while the filament is wide enough to allow for the silicone oil to flow towards the tip. Close to pinch-off the filament is already too thin for this. Finger pinch-off takes place at the junction of the filament and the droplet. Given that before and after pinch-off, droplets are by large the main contribution to the variation of $R_{g}$, the argument of the preceding section means that the typical area of a droplet should be proportional to $1 / n_{m}^{*}$, where $n_{m}^{*}$ is the number of fingers in the pattern. Accordingly, the overall area of all the droplets should be a constant, irrespective of the number of droplets. This is actually an average description, since not all droplets in a given experiment are identical, and there is some dispersion of their areas.

We have measured the total area covered by the first generation of emitted droplets (the contribution of subsequent generations is negligible) in a large collection of experiments with different experimental parameters $\left(b, R_{0}, \Omega\right)$, giving rise to a wide distribution of numbers of fingers. The results are presented in Fig. 18, where the total area of the droplets is plotted against $\pi R_{0}^{2}$, the area of the initial circle. The graph demonstrates clearly that the total area covered by the droplets is essentially a constant, as predicted, rather than a fraction or some other function of the initial area. This constancy holds above a finite threshold $R_{0 c} \simeq 35 \mathrm{~mm}$ $\left(\pi R_{0 c}^{2} \simeq 4000 \mathrm{~mm}^{2}\right)$. We have not found a limit to this behavior at large radii.

The threshold value $R_{0 c} \simeq 35 \mathrm{~mm}$ is consistent with the fact that the overall droplet area $\left(A_{0} \simeq 1200 \mathrm{~mm}^{2}\right)$ would correspond to an initial circle of radius $R_{0} \simeq 20 \mathrm{~mm}$. Thus, for initial conditions with $R_{0}<20 \mathrm{~mm}$ the constancy of the overall droplet area cannot hold. For $20 \mathrm{~mm}<R_{0}<35 \mathrm{~mm}$ the constant droplet area is not yet attained because the fingers also carry a fraction of the overall available area.

The large error bars in the plot reflect that the spatial resolution of our images is relatively low to measure the area of droplets, since their radii fall in the range from 2 to $7 \mathrm{~mm}$. The number of pixels on the droplet interface is comparable to the number of pixels within the droplet. The error bars account for the dispersion in droplet area after considering or neglecting the contribution of the droplet interface. Another error source is a slight (but detectable) increase of the droplet area as the droplet velocity increases. This effect is due to the thickening of the vaseline films coating the two glass plates, above and below a silicone droplet, compressing the droplet in the vertical direction and expanding it in the horizontal plane. To minimize this error source we have measured the droplet area well beyond pinch-off, when all droplets move at roughly the same velocity.

The numerical values $R_{0 c} \simeq 35 \mathrm{~mm}$ and droplet area $A_{0}$ $=1200 \mathrm{~mm}^{2}$ would presumably change if surface tension and/or viscosity contrast were changed. Theoretical analysis and numerical simulations that we are currently performing show that surface tension and viscosity contrast do play a role in the pinch-off process, e.g., in the time to pinch-off and in the mass flow through the filaments. Surface tension and viscosity contrast may also have an influence on pinchoff through three-dimensional effects not considered in the Hele-Shaw equations. This can modify the mass flow into the droplets, thus changing $A_{0}$ (and hence $R_{0 c}$ ). We expect this change to be small, however, since this flow is already low well before pinch-off takes place. Additional experiments would be necessary to ascertain the relevance of these effects.

\section{F. The long-time regime after pinch-off}

Once the silicone fingers experience the first generation of pinch-off, the dynamics of the patterns is substantially modified. After pinching, the silicone oil filaments, still connected to the central region, are too thin to allow for a substantial outward flux of silicone oil. Thus, the pattern after pinch-off is rather stable, and it takes a long time to displace it outwards. The pattern must reorganize itself to emit the remaining silicone oil. We recall that the mechanisms for it, described in Sec. II D, are: (i) the generation of new silicone oil fingers in a nonsystematic way and (ii) the coalescence of incoming vaseline oil fingers, which allows a region of silicone oil to become disconnected from the center and be emitted outwards. In the last stages of the pattern the vaseline fingers become considerably wide, and their number is 

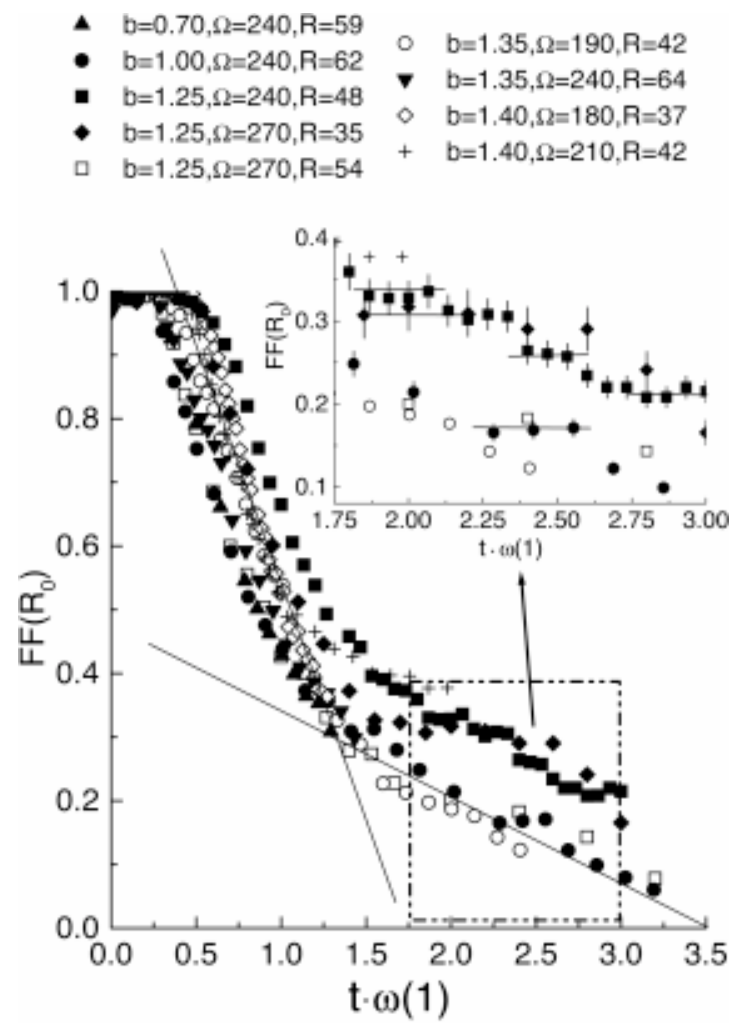

FIG. 19. The fraction of the initial circle filled with silicone oil, $F F\left(R_{0}, t\right)$, as a function of the dimensionless time $t \cdot \omega(1)$. The straight lines are drawn to help recognizing a change in the rate of silicone oil emission, in the nonlinear regime. The inset shows the presence of flat regions in the latest stages. The values of $b, \Omega$, and $R$ are given in $\mathrm{mm}$, rpm, and $\mathrm{mm}$ respectively.

reduced dramatically down to values between two and five fingers. They remain separated by rather thin silicone oil filaments, which finally break up in many minute droplets.

With the purpose of making a characterization of this long-time regime, we introduce the parameter $F F(r, t)$ (filling fraction) defined as

$$
F F(r, t)=\frac{\int_{0}^{2 r} r^{\prime} d r^{\prime} \int_{0}^{2 \pi} f\left(r^{\prime}, \phi\right) d \phi}{\int_{0}^{r} r^{\prime} d r^{\prime} \int_{0}^{2 \pi} d \phi},
$$

where

$$
f(r, \phi)= \begin{cases}1 & \text { silicone oil } \\ 0 & \text { vaseline oil } .\end{cases}
$$

$F F(r, t)$ measures the ratio of the area occupied by the silicone oil to the total area, in a circle of radius $r$ at time $t$. We focus on $F F\left(R_{0}, t\right)$, corresponding to a circle defined by the initial condition of the pattern. We study how the silicone oil initially filling this circle is displaced outwards, particularly at long times.

Figure 19 shows the evolution of $F F\left(R_{0}, t\right)$ as a function of the dimensionless time $t \cdot \omega(1)$ in different experiments. As usual, there is first a latency period in which the initial condition is not modified, followed by the exponential change characteristic of the linear regime during a very short time, and then a decay associated with the nonlinear regime. This decay comprises two stages: one at a nearly constant high rate, followed by another at much lower rate and punctuated by constant $F F$ intervals (inset), corresponding to the long-time regime analyzed in this section.

The transition from one stage to the other, for one particular experiment, is illustrated in the figure by two straight lines drawn to guide the eye. The transition is much more abrupt in experiments with small $R_{0}$ and few fingers (15, say) than in experiments with large $R_{0}$ and many fingers (70, say). There are two reasons for this: first, the mass emitted outwards in the first stages of the nonlinear regime is roughly constant for all experiments, and therefore the experiments with larger $R_{0}$ have more silicone oil to emit in the last stages. Second, in the experiments with a larger number of fingers the silicone oil fingers present an irregular, filamentlike appearance, which favors the main mechanisms of silicone oil emission (reconnection of vaseline oil fingers, elongation of the silicone oil finger necks). On the contrary, the experiments with smaller $R_{0}$ and few fingers present a more regular arrangement in the mixing zone, with vaseline oil fingers clearly separated by silicone oil fingers. Being so, inner reconnection is not easy, nor is the elongation of silicone oil finger necks. These difficulties prevent the system from displacing the silicone oil outwards, once the thicker silicone oil finger has pinched. Consequently, the rate at which $F F\left(R_{0}, t\right)$ decays is abruptly reduced.

Another interesting feature of $F F\left(R_{0}, t\right)$ in the latest stages of the nonlinear regime is the presence of plateaus, i.e., periods where the filled fraction does not change appreciably, showing that there is no flux of silicone oil through the circumference of radius $R_{0}$. The plateaus can be clearly recognized in the inset of Fig. 19. Their presence does not mean that the interface is frozen. Suppose that new fingers have been formed at time $t$, which account for the flux of silicone oil through $R_{0}$ at time $t$. A plateau starts when these fingers, before pinching, become too narrow to allow for any flux across $R_{0}$. Furthermore, immediately after the fingers pinching the filaments left behind relax, allowing no flux to cross $R_{0}$ until new fingers grow again from the radius they were formed from and reach the radius $R_{0}$.

In summary, the last stages of the highly nonlinear regime are particularly lengthy. The outward displacement of the silicone oil in these last stages relies on the asymmetries of the pattern, and is dominated by the inner reconnection of incoming vaseline fingers and the pinch-off of new outgoing silicone fingers.

\section{G. The role of low surface tension}

Having a low viscosity contrast implies working with two liquids, which in our case results in a very low value of the interfacial tension $\sigma$, about 1 order of magnitude smaller than at an air-liquid interface. The question arises as whether the dynamic and morphological features identified in our work are related only to the low viscosity contrast, or the low interfacial tension plays also a relevant role. In this sense, it is important to note that the morphologies observed in the present experiments are remarkably similar to those obtained numerically for miscible flows (negligible interfacial tension) in a rotating Hele-Shaw cell. ${ }^{19}$ 

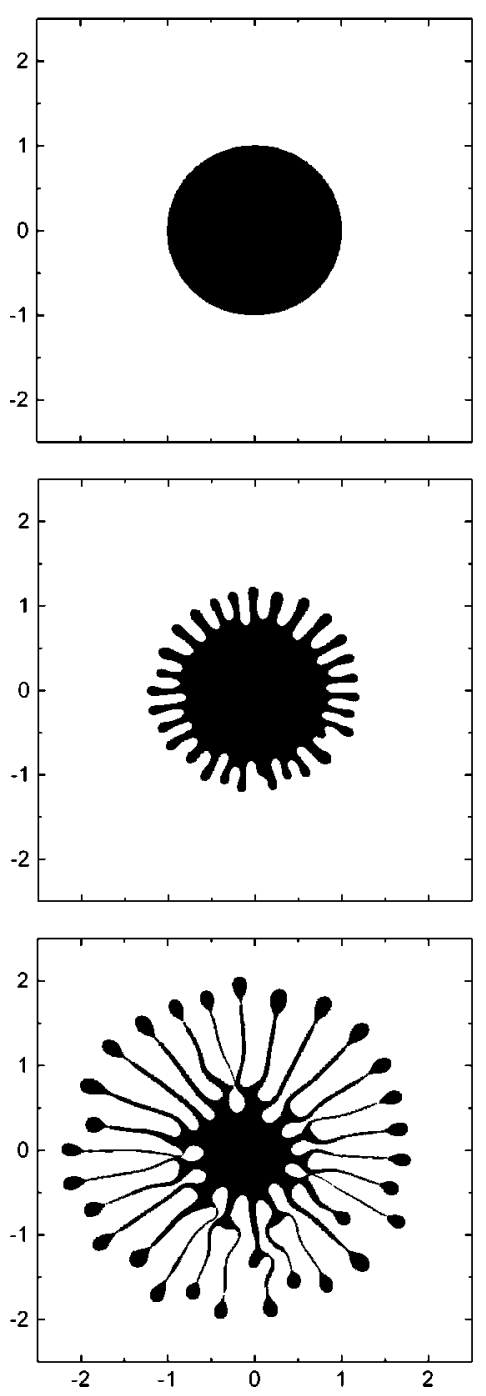
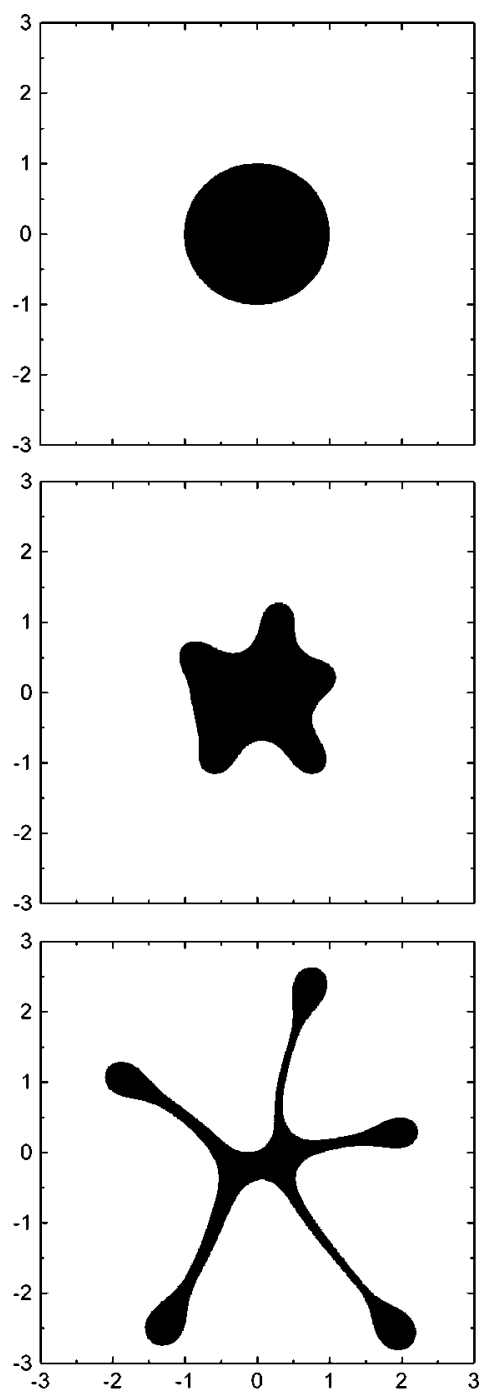

FIG. 20. Numerical integration of the spatiotemporal evolution of a circular drop. The three snapshots on the left correspond to $A=0.4$ and $S=2500$, and the three snapshots on the right to $A=0.4$ and $S=80$. The interfaces are shown at dimensionless times $\tilde{t}=0$, $2 \times 10^{-4}, 4.4 \times 10^{-4}$ (left), and $\tilde{t}=0,2.5 \times 10^{-2}, 3.7$ $\times 10^{-2}$ (right), measured in units of $12\left(\mu_{1}\right.$ $\left.+\mu_{2}\right) R_{0}^{3} /\left(b^{2} \sigma\right)$.
Viscosity contrast and interfacial tension could be modified nearly independently in channel geometry, by controlling the temperature of a binary fluid mixture near criticality. ${ }^{4}$ Even though our current experimental setup does not allow us to work along this way, we will present here accurate numerical solutions of our flows that do provide a definite answer to the question above-together with interesting additional information.

Figure 20 presents the results of two numerical experiments. The one on the left is for $A=0.4, S=2500$, and the one on the right for $A=0.4, S=80$. The same initial condition has been used in both runs: a perfect circle perturbed with 50 modes of $0.1 \%$ amplitude and random phases. The numerical algorithm is described in detail in Ref. 20.

The value $A=0.4$ has been chosen to match the viscosity contrast in the experiments. This value is representative of low viscosity contrast flows because, as mentioned in Sec. II C, the fingering dynamics is sensitive to $A$ only when $A$ is very close to 1 (around 0.9 ), and practically insensitive to $A$ in the remaining interval of lower $A .^{3,5,6}$ We have performed the same simulations for $A=0$ and obtained numerical results nearly identical to the ones presented in Fig. 20.

The point to stress is that the simulation presented in the left panel of the figure, purposely designed to produce a large number of fingers, reproduces the basic features observed in our experiments: (i) All fingers formed in the early stages of the instability keep growing in the deeply nonlinear regime, demonstrating the absence of finger competition. (ii) As in experiments, the number of fingers matches the wave number of the fastest growing mode in the linear dispersion relation, $n_{m}=\sqrt{(1+S) / 3} \simeq 29$. (iii) Competition for space of the incoming fingers gives rise, in the later stages, to a typical fork-like structure in which the tip of an outcoming finger bifurcates in two new fingers. (iv) Droplets form at the finger ends, and characteristic signs of imminent pinch-off at the finger-droplet junctions are visible, just before the code is halted due to lack of accuracy.

It is worth noting that the same spatio-temporal evolution of the interface (in dimensionless variables) is obtained for all sets of parameters compatible with given values of $A$ (viscosity contrast) and $S$ (inverse dimensionless surface tension), because these are the only two dimensionless parameters of the problem for $Q=0 .^{7}$ In particular, a modification of the surface tension, $\sigma$, has the only effect of changing the time scale, if the other parameters of the flow are modified in 
such a way that $S$ remains unchanged. This means that the results presented in Fig. 20 are representative of the dynamics for the $A, S$ values reported, irrespective of the actual value of $\sigma$, and therefore the features listed above are specific of low viscosity contrast flows, and cannot be attributed to the low value of $\sigma$ in the experiments.

A separate issue refers to the validity of the dynamic equations for extreme values of the physical parameters. For instance, a low value of $\sigma$ may lead to morphological features on the scale of the cell thickness $b$. Even with this concern in mind, the striking similarity with the numerical simulations makes us confident that our experiments are properly described by the Hele-Shaw equations.

In the right panel of Fig. 20 we show the spatio-temporal evolution for low viscosity contrast fingering (small $A$ ) with a reduced number of fingers (small $S$ ). Let us recall that these patterns are not accessible in our experiments because $\sigma$ is small and $\Omega$ must be kept relatively large for the instability to develop before dripping in the vertical direction sets in. These simulations show again that fingers do not compete (they all keep growing). In this case the absence of competition cannot be attributed solely to the low viscosity contrast, but predominantly to the increasing available space for outgrowing fingers in the radial geometry. Nevertheless, numerical simulations in the channel geometry ${ }^{6}$ show the same lack of competition for similar number of fingers than the two sets of parameters of Fig. 20.

\section{SUMMARY AND CONCLUSIONS}

We have conducted a systematic investigation of the dynamics and morphology of the viscous fingering patterns formed in a rotating Hele-Shaw cell, due to the displacement of a lighter fluid by a denser fluid of comparable dynamic viscosity.

Working with two liquids has been found to be particularly involved and delicate. The liquids must be introduced in the cell under well controlled conditions. When displaced by the second liquid, the first liquid leaves a coating layer on each glass plate whose thickness depends on the injection rate during the preparation. The experiment must also be carried out before dripping of these coating layers sets in. Great care must also be put in avoiding air bubbles at the interface between the two liquids.

The patterns obtained in our experiments make it evident that the condition of low viscosity contrast between the two liquids suppresses the dynamic competition between fingers, characteristic of air-liquid displacements (high viscosity contrast). The morphologies observed are strikingly different from previous viscous fingering morphologies found in radial geometry. Instead of being unstable against tip-splitting or side-branching, the fingers here stretch along the radial direction and form a droplet at the tip, which usually detaches from the finger through a pinch-off singularity.

The radial geometry imposes a modification of the usual Young-Laplace boundary condition at the interface (pressure jump proportional to interface curvature), which introduces a dependence of the linear dispersion relation on gap spacing. We have presented experimental evidence that this effect is relevant and measurable on displacements that involve a large number of fingers. This is the most frequent situation in our experiments. The delicate scrutiny of the linear dispersion relation required to verify this effect has been possible thanks to the lack of dynamic competition between fingers, characteristic of low viscosity contrast displacements, which allows us to equate the number of fingers in the nonlinear regime to the fastest growing wave number in the linear regime.

Concerning the empirical scaling of different characteristic measures of the patterns, we have found that most measures follow the time scale $t \cdot \omega(1)$, but the interface stretching follows the time scale $t \cdot \omega(1) \cdot n_{m}^{*}$, which introduces the number $S$ (ratio of centrifugal to capillary forces) in the scaling. The same happened also for high viscosity contrast displacements. ${ }^{9}$ Nevertheless, one must be careful in drawing conclusions from the comparison between our experiments and those of Ref. 9 (for high viscosity contrast), because the latter were carried out in a dry cell, and hence under very different wetting conditions. By comparing experimental results to numerical simulations of air-oil displacements, we have found that the Hele-Shaw equations reproduce only the experiments of oil displacing air when the cell has been prewetted. Wetting conditions play a determinant role in the pattern morphologies, and may also be relevant in the scaling of the different measures. This issue will be addressed in a forthcoming work.

The problem of finger pinch-off and the formation of droplets is interesting for a variety of reasons. Pinch-off seems to be favored by the low viscosity contrast and by the rotation of the cell (since centrifugal pressure increases linearly with radial distance to the rotation axis). But the question remains whether the finite-time pinch-off observed in our experiments is only a three-dimensional effect not contained in the two-dimensional Hele-Shaw equations (which could lead to pinch-off at infinite time) or, on the contrary, the two-dimensional model can lead spontaneously to finitetime pinch-off. The question cannot be answered experimentally, since in experiments the three-dimensional structure of the meniscus shows up unavoidably near pinch-off, as soon as the width of a finger becomes comparable to the gap spacing $b$. The interplay between viscosity contrast and rotation in the possible existence of finite-time singularities in the two-dimensional Hele-Shaw equations is an interesting open question.

\section{ACKNOWLEDGMENTS}

We are grateful to Ll. Carrillo and E. Pauné for enlightening discussions. The technical support of M. Castro, M. del Río, J. Llorens (Servei de Reologia, UB), and F. Comelles (IIQA, CSIC), is greatly appreciated. This research is partially supported by the DGES (Spain), Project No. BXX-2000-0638-C02-02, the European Commission through Network No. HPRN-CT-2002-00312, and by the DURSI (Generalitat de Catalunya), Project No. 2001-SGR-00433. E.A.L. and J.O. acknowledge additional financial support from the DURSI. 
${ }^{1}$ M. C. Cross and P. C. Hohenberg, "Pattern formation outside of equilibrium,” Rev. Mod. Phys. 65, 851 (1993).

${ }^{2}$ P. Pelcé, Dynamics of Curved Fronts, Perspectives in Physics (Academic, San Diego, CA, 1988).

${ }^{3}$ G. Tryggvason and H. Aref, "Numerical experiments on Hele-Shaw flow with a sharp interface," J. Fluid Mech. 136, 1 (1983); "Fingering interaction mechanism in stratified Hele-Shaw flow," 154, 287 (1985); J. Casademunt, D. Jasnow, and A. Hernández-Machado, "Interface equation and viscosity contrast in Hele-Shaw flow," Int. J. Mod. Phys. B 6, 1647 (1992).

${ }^{4}$ J. V. Maher, "Development of viscous fingering patterns," Phys. Rev. Lett. 54, 1498 (1985); M. W. DiFrancesco and J. V. Maher, "Noisy and regular features in Saffman-Taylor patterns," Phys. Rev. A 39, 4709 (1989); "Hele-Shaw scaling properties of low contrast Saffman-Taylor flows," 40, 295 (1989); H. Zhao and J. V. Maher, "Viscous fingering experiments with periodic boundary conditions," ibid. 42, 5894 (1990).

${ }^{5}$ J. Casademunt and D. Jasnow, "Defects dynamics in viscous fingering," Phys. Rev. Lett. 67, 3677 (1991); "Finger competition and viscosity contrast in viscous fingering. A topological approach," Physica D 79, 387 (1994).

${ }^{6}$ E. Pauné, "Interface dynamics in two-dimensional viscous flows," Ph.D. thesis, University of Barcelona, Barcelona, Spain, 2002.

${ }^{7}$ E. Alvarez-Lacalle, J. Casademunt, and J. Ortín, "Systematic weakly nonlinear analysis of interfacial instabilities in Hele-Shaw flows," Phys. Rev. E 64, 016302 (2001); "Systematic weakly nonlinear analysis of radial viscous fingering," 68, 026308 (2003).

${ }^{8}$ L. W. Schwartz, "Instabilities and fingering in a rotating Hele-Shaw cell or porous medium," Phys. Fluids A 1, 167 (1989).

${ }^{9}$ Ll. Carrillo, F. X. Magdaleno, J. Casademunt, and J. Ortín, "Experiments in a rotating Hele-Shaw cell," Phys. Rev. E 54, 6260 (1996).

${ }^{10}$ V. M. Entov, P. I. Etingof, and D. Ya. Kleinbock, "On nonlinear interface dynamics in Hele-Shaw flows,” Eur. J. Appl. Math. 6, 399 (1996).

${ }^{11}$ D. Crowdy, "Theory of exact solutions for the evolution of a fluid annulus in a rotating Hele-Shaw cell," Q. Appl. Math. 60, 11 (2002); "On a class of geometry-driven free boundary problems," SIAM (Soc. Ind. Appl. Math.) J. Appl. Math. 62, 945 (2001).

${ }^{12}$ F. X. Magdaleno, A. Rocco, and J. Casademunt, "Interface dynamics in Hele-Shaw flows with centrifugal forces: Preventing cusp singularities with rotation," Phys. Rev. E 62, R5887 (2000).

${ }^{13}$ Ll. Carrillo, J. Soriano, and J. Ortín, "Radial displacement of a fluid annulus in a rotating Hele-Shaw cell," Phys. Fluids 11, 778 (1999); "Interfacial instabilities of a fluid annulus in a rotating Hele-Shaw cell," 12, 1685 (2000)

${ }^{14}$ L1. Carrillo, "Estudio experimental de sistemas fuera del equilibrio," Ph.D. thesis, University of Barcelona, Barcelona, Spain, 2000.

${ }^{15}$ C. W. Park and G. M. Homsy, "Two-phase displacements in Hele-Shaw cells: Theory," J. Fluid Mech. 139, 291 (1984).

${ }^{16}$ L. Schwartz, "Stability of Hele-Shaw flows: The wetting-layer effect," Phys. Fluids 29, 3086 (1986).

${ }^{17}$ T. Maxworthy, "Experimental study of interface instability in a HeleShaw cell," Phys. Rev. A 39, 5863 (1989).

${ }^{18}$ K. V. McCloud and J. V. Maher, "Experimental perturbation to SaffmanTaylor flow," Phys. Rep. 260, 139 (1995).

${ }^{19}$ C.-Y. Chen and S.-W. Wang, "Interfacial instabilities of miscible fluids in a rotating Hele-Shaw cell,” Fluid Dyn. Res. 30, 315 (2002).

${ }^{20}$ E. Pauné, M. Siegel, and J. Casademunt, "Effects of small surface tension in Hele-Shaw multifinger dynamics: An analytical and numerical study," Phys. Rev. E 66, 046205 (2002). 